\title{
A new conformable nabla derivative and its application on arbitrary time scales
}

\author{
Mohamad Rafi Segi Rahmat ${ }^{1 *}$ (D) and M. Salmi M. Noorani²
}

\section{"Correspondence:}

mohd.rafi@nottingham.edu.my 'School of Mathematical Sciences, University of Nottingham Malaysia, Jalan Broga, Semenyih, Selangor Darul Ehsan 43500, Malaysia Full list of author information is available at the end of the article

\begin{abstract}
In this article, we introduce a new type of conformable derivative and integral which involve the time scale power function $\widehat{\mathcal{G}}_{\eta}(t, a)$ for $t, a \in \mathbb{T}$. The time scale power function takes the form $(t-a)^{\eta}$ for $\mathbb{T}=\mathbb{R}$ which reduces to the definition of conformable fractional derivative defined by Khalil et al. (2014). For the discrete time scales, it is completely novel, where the power function takes the form $(t-a)^{(\eta)}$ which is an increasing factorial function suitable for discrete time scales analysis. We introduce a new conformable exponential function and study its properties. Finally, we consider the conformable dynamic equation of the form $\nabla_{a}^{\gamma} y(t)=y(t, f(t))$, and study the existence and uniqueness of the solution. As an application, we show that the conformable exponential function is the unique solution to the given dynamic equation. We also examine the analogue of Gronwall's inequality and its application on time scales.
\end{abstract}

Keywords: Fractional calculus; Calculus on time scales; Conformable nabla derivative; Conformable nabla integral

\section{Introduction}

Fractional calculus becomes an important area of research in mathematical analysis and applications. Various definition of fractional derivative operators such as RiemannLiouville, Caputo, Grünwald-Letnikov, and Erdélyi-Kober to mention few, were introduced and successfully applied to solve complex systems in science and engineering (see $[1-3])$. However, the semigroup properties of these fractional operators behave well in some cases only.

In 2014, Khalil et al. [4] introduced a new simple well-behaved definition of the fractional derivative called conformable fractional derivative $T_{\alpha} f(t)(\alpha \in(0,1])$ of a function $f: \mathbb{R}^{+} \rightarrow \mathbb{R}$ at $t>0$ which depends on the basic limit definition of the classical derivative. This new fractional derivative definition has attracted much attention in recent years. In 2015, Abdeljawad [5] made an extensive research of the newly introduced conformable fractional calculus. In his work, he generalizes the definition of conformable fractional derivative $T_{\alpha}^{a} f(t)$ for $t>a \in \mathbb{R}^{+}$as

$$
T_{\alpha}^{a} f(t):=\lim _{\epsilon \rightarrow 0} \frac{f\left(t+\epsilon(t-a)^{1-\alpha}\right)-f(t)}{\epsilon}
$$

(c) The Author(s) 2021. This article is licensed under a Creative Commons Attribution 4.0 International License, which permits use sharing, adaptation, distribution and reproduction in any medium or format, as long as you give appropriate credit to the original author(s) and the source, provide a link to the Creative Commons licence, and indicate if changes were made. The images or other third party material in this article are included in the article's Creative Commons licence, unless indicated otherwise in a credit line to the material. If material is not included in the article's Creative Commons licence and your intended use is not permitted by statutory regulation or exceeds the permitted use, you will need to obtain permission directly from the copyright holder. To view a copy of this licence, visit http://creativecommons.org/licenses/by/4.0/. 
In this case, if the function $f(t)$ is differentiable, then

$$
T_{\alpha}^{a} f(t)=(t-a)^{1-\alpha} f^{\prime}(t)
$$

It is clear that if $\alpha=1$, we have $T_{1}^{a} f(t)=\lim _{\epsilon \rightarrow 0} \frac{f(t+\epsilon)-f(t)}{\epsilon}=f^{\prime}(t)$.

The conformable fractional integral of a function $f(t)$ from $a \in \mathbb{R}^{+}$is defined by

$$
I_{\alpha}^{a} f(t):=\int_{a}^{t}(s-a)^{\alpha-1} f(s) d s, \quad 0<\alpha \leq 1 .
$$

Note that for $a=0$, we have $T_{\alpha}^{0} f(t)=T_{\alpha} f(t)$ and $I_{\alpha}^{0} f(t)=I_{\alpha} f(t)$, as suggested in [4].

An important reason for its introduction is specified by the fact that this derivative has many well-known properties of integer-order derivatives, among which we can highlight Rolle's theorem, mean value theorem, product rule, quotient rule, chain rule, fractional power series expansion, and fractional Laplace transform (see [4-7]), which the classical fractional derivatives do not obey. To further justify the introduction of the conformable fractional derivative and its consequences, we refer to [8].

Since then, the theory of conformable fractional calculus and its applications have been studied by many authors (see, for instance, [9-15]). Chung [16] used the conformable fractional derivative and integral to discuss fractional Newtonian mechanics and Rezazadeh et al. [17] investigated the stability of linear conformable fractional systems from the point view of control theory (see also [18]). It is worth noting that the conformable fractional derivative does not have a physical meaning as the Riemann-Liouville or Caputo derivatives.

In [19] Anderson and Ulness made a remark that the discussed derivative is local in nature and hence the correct name must be "conformable derivative" instead as introduced "conformable fractional derivative." It is worth mentioning here the criticism made about the definition of conformable fractional derivative by the authors of [20,21].

On the other hand, Benkhettou et al. [22] extended the definition of (conformable) fractional derivative to arbitrary time scales. Meanwhile, in [23], the authors have studied a version of the nabla conformable fractional derivative on arbitrary time scales. Namely, for a function $f: \mathbb{T} \rightarrow \mathbb{R}$, the nabla conformable fractional derivative, $T_{\nabla, \alpha} f(t) \in \mathbb{R}$ of order $\alpha \in(0,1]$ at $t \in \mathbb{T}_{\kappa}$ and $t>0$, was defined as follows.

For any $\epsilon>0$, there exists a neighborhood $U=(t-\delta, t+\delta) \cap \mathbb{T}$ for some $\delta>0$ such that

$$
\mid\left[f(\rho(t)-f(s)] t^{1-\alpha}-T_{\nabla, \alpha}(f)(t)[\rho(t)-s]|\leq \epsilon| \rho(t)-s \mid \quad \text { for all } s \in U\right. \text {. }
$$

The nabla conformable fractional integral is defined by

$$
\int f(t) \nabla_{\alpha} t:=\int f(t) t^{\alpha-1} \nabla t, \quad 0<\alpha \leq 1
$$

Further development on conformable fractional derivative and its applications on arbitrary time scales can be seen through the articles [24-32].

Motivated by the results in [5], we generalize the definition of the nabla conformable fractional derivative and integral on time scales in [23] by replacing $(t-a)^{1-\alpha}$ with a generalized time scale power function $\widehat{\mathcal{G}_{1-\gamma}}(t, a)$. With the new definition of the nabla conformable derivative, we are able to define the conformable exponential function and study 
its properties. We also prove an analogue of Gronwall's inequality which will be useful in establishing the stability of the nabla conformable fractional dynamical systems on time scales.

\section{Preliminaries}

In this section, we review some basic concepts and notations of calculus of time scales used in this article. The reader interested in the subject of time scales is referred to [33-35].

A time scale $\mathbb{T}$ is an arbitrary closed nonempty subset of $\mathbb{R}$. The forward and backward jump operators $\sigma, \rho: \mathbb{T} \rightarrow \mathbb{T}$ are defined by

$$
\sigma(t):=\inf \{s \in \mathbb{T} \mid s>t\} \quad \text { and } \quad \rho(t):=\sup \{s \in \mathbb{T} \mid s<t\}
$$

respectively. The forward and backward graininess functions $\mu, v: \mathbb{T} \rightarrow[0, \infty)$ are defined by

$$
\mu(t):=\sigma(t)-t \quad \text { and } \quad v(t):=t-\rho(t)
$$

respectively. We say that $t \in \mathbb{T}$ is right-scattered (resp., left-scattered) if $\sigma(t)>t$ (resp., $\rho(t)<t)$. Points that are right- and left-scattered at the same time are called isolated. Also, if $t<\sup \mathbb{T}$ and $\sigma(t)=t$, then $t$ is called right-dense, and if $t>\inf \mathbb{T}$ and $\rho(t)=t$, then $t$ is called left-dense. If $\mathbb{T}$ has a right-scattered minimum $m$, then $\mathbb{T}_{\kappa}=\mathbb{T}-\{m\}$. Otherwise, $\mathbb{T}_{\kappa}=\mathbb{T}$.

Throughout this paper we assume that $a, b \in \mathbb{T}$ and $a<b$. We then define the interval $[a, b]$ in $\mathbb{T}$ by $[a, b]_{\mathbb{T}}:=\{t \in \mathbb{T} \mid a \leq t \leq b\}$. Open intervals and half-open intervals are defined analogously.

We assume throughout that a time scale $\mathbb{T}$ has the topology that it inherits from the real numbers with the standard topology. For $f: \mathbb{T} \rightarrow \mathbb{R}$ and $t \in \mathbb{T}_{\kappa}$, define the nabla derivative of $f(t)$, denoted $f^{\nabla}(t)$, to be the number (provided it exists) with the property that given any $\epsilon>0$, there is a neighborhood $\mathcal{U}$ of $t$ (i.e., $\mathcal{U}_{t}=(t-\delta, t+\delta) \cap \mathbb{T}$ for some $\delta>0$ ) such that

$$
\left|f(\rho(t))-f(s)-f^{\nabla}(t)(\rho(t)-s)\right| \leq \epsilon|\rho(t)-s|
$$

for all $s \in U$. For $\mathbb{T}=\mathbb{R}$, we have $f^{\nabla}=f^{\prime}$, the usual derivative, and for $\mathbb{T}=\mathbb{Z}$ we have the backward difference operator, $f^{\nabla}(t)=\nabla f(t):=f(t)-f(t-1)$.

A function $f: \mathbb{T} \rightarrow \mathbb{R}$ is left-dense continuous (ld-continuous) provided it is continuous at left-dense points in $\mathbb{T}$ and its right-sided limits exist (finite) at right-dense point in $\mathbb{T}$. The set of all ld-continuous functions $f: \mathbb{T} \rightarrow \mathbb{R}$ will be denoted by $C_{\mathrm{ld}}(\mathbb{T})$. Similarly, the set of all ld-continuous and nabla differentiable functions will be denoted by $C_{\mathrm{ld}}^{\nabla}(\mathbb{T})$.

Theorem 2.1 ([34]) Assume $f: \mathbb{T} \rightarrow \mathbb{R}$ is a function and let $t \in \mathbb{T}_{\kappa}$. Then we have:

(i) Iff is nabla differentiable at $t$, the $f$ is continuous at $t$.

(ii) If $f$ is continuous at a left-scattered $t$, the $f$ is nabla differentiable at $t$ with

$$
f^{\nabla}(t)=\frac{f(t)-f(\rho(t))}{v(t)} .
$$


(iii) Iff is left-dense, then $f$ is nabla differentiable at $t$ if and only if the limit

$$
\frac{f(t)-f(s)}{t-s}
$$

exists as a finite number. In this case

$$
f^{\nabla}(t)=\frac{f(t)-f(s)}{t-s} .
$$

A function $F: \mathbb{T} \rightarrow \mathbb{R}$ is called a nabla antiderivative of $f: \mathbb{T} \rightarrow \mathbb{R}$, provided that $F^{\nabla}(t)=$ $f(t)$ holds for all $t \in \mathbb{T}_{\kappa}$, then the nabla integral of $f$ is defined by

$$
\int_{a}^{b} f(t) \nabla t=F(b)-F(a)
$$

It follows that, if $f \in C_{\mathrm{ld}}(\mathbb{T})$ and $t \in \mathbb{T}_{\kappa}$, then

$$
\int_{\rho(t)}^{t} f(\tau) \nabla \tau=[t-\rho(t)] f(t)
$$

Note that if $|f(t)| \leq g(t)$ on $[a, b)$, then

$$
\left|\int_{a}^{b} f(t) \nabla t\right| \leq \int_{a}^{b} g(t) \nabla t
$$

Let $f: \mathbb{T} \times \mathbb{T} \rightarrow \mathbb{R}$ be a function and denote the nabla derivative of $f(t, s)$ with respect to $t$ (for fixed $s$ ) by $f^{\nabla}(t, s)$. If $f$ and $f^{\nabla}$ are continuous, then

$$
\left[\int_{a}^{t} f(t, s) \nabla s\right]^{\nabla}=f(\rho(t), t)+\int_{a}^{t} f^{\nabla}(t, s) \nabla s .
$$

Now, we remind further aspects of time scale calculus, which will be needed later; see, e.g., [34].

Definition 2.2 Let $\mathbb{T}$ be a time scale. A function $f: \mathbb{T} \times \mathbb{R} \rightarrow \mathbb{R}$ is called

(i) ld-continuous, if $g$ defined by $g(t):=f(t, y(t))$ is ld-continuous for any ld-function $y: \mathbb{T} \rightarrow \mathbb{R}$.

(ii) bounded on a set $S \subset \mathbb{T} \times \mathbb{R}$, if there exists a constant $M>0$ such that

$$
\mid f(t, y(t) \mid \leq M \quad \text { for all }(t, y) \in S
$$

(iii) Lipschitz continuous on a set $S \subset \mathbb{T} \times \mathbb{R}$, if there exists a constant $L>0$ such that

$$
\left|f\left(t, y_{1}\right)-f\left(t, y_{2}\right)\right| \leq L\left|y_{1}-y_{2}\right| \quad \text { for all }\left(t, y_{1}\right),\left(t, y_{2}\right) \in S
$$

The following lemma is a nabla version of the Proposition 2.6 in [36]. 
Lemma 2.3 Suppose $\mathbb{T}$ is a time scale and $f$ is an increasing ld-continuous function on the interval $[a, b]_{\mathbb{T}}$. If $\tilde{f}$ is the extension off to the real interval $[a, b]$ given by

$$
\tilde{f}(s)= \begin{cases}f(s), & \text { if } s \in \mathbb{T} ; \\ f(t), & \text { if } s \in(\rho(t), t) \not \subset \mathbb{T},\end{cases}
$$

then

$$
\int_{a}^{b} f(\rho(t)) \nabla t \leq \int_{a}^{b} \tilde{f}(t) d t .
$$

Proof Let $r \in[a, b]_{\mathbb{T}}$ be a left-scattered point. Then we have

$$
\int_{\rho(r)}^{r} f(t) \nabla t=[r-\rho(r)] f(r) .
$$

Since $f$ is an increasing function, its extension $\tilde{f}$ will be an increasing continuous function. Thus, the mean value theorem for integrals implies

$$
[r-\rho(r)] \tilde{f}(\rho(r)] \leq \int_{\rho(r)}^{r} \tilde{f}(t) d t \leq[r-\rho(r)] \tilde{f}(r)
$$

and

$$
[r-\rho(r)] f(\rho(r)] \leq \int_{\rho(r)}^{r} \tilde{f}(t) d t \leq[r-\rho(r)] f(r) .
$$

Hence, we have

$$
\int_{\rho(r)}^{r} f(\rho(t)) \nabla t \leq \int_{\rho(r)}^{r} \tilde{f}(t) d t
$$

Suppose, if $[a, b]$ has only one left-scattered point $s$, then we have

$$
\begin{aligned}
\int_{a}^{b} f(\rho(t)) \nabla t & =\int_{a}^{\rho(s)} f(\rho(t)) \nabla t+\int_{\rho(s)}^{s} f(\rho(t)) \nabla t+\int_{s}^{b} f(\rho(t)) \nabla t \\
& \leq \int_{a}^{\rho(s)} \tilde{f}(t) d t+\int_{\rho(s)}^{s} \tilde{f}(t) d t+\int_{s}^{b} \tilde{f}(t) d t
\end{aligned}
$$

If we repeat the above steps for $n$ left-scattered points in $[a, b]$, we obtain

$$
\int_{a}^{b} f(\rho(t)) \nabla t \leq \int_{a}^{b} \tilde{f}(t) d t
$$

This completes the proof.

Next, we introduce the generalized time scale power function $\mathcal{G}_{n}(t, s) \in \mathbb{R}^{+}$for $n \in \mathbb{N}_{0}$ and $s, t \in \mathbb{T}$. 
Definition 2.4 Let $[s, t] \subset \mathbb{T}$ and $s<t$. The generalized time scale power function $\mathcal{G}_{n}$ : $\mathbb{T} \times \mathbb{T} \rightarrow R^{+}$for $n \in \mathbb{N}_{0}$ is defined by

$$
\widehat{\mathcal{G}}_{n}(t, s)= \begin{cases}(t-s)^{n}, & \text { if }[s, t] \text { dense; } \\ \prod_{j=0}^{n-1}\left(t-\rho^{j}(s)\right), & \text { if }[s, t] \text { isolated }\end{cases}
$$

and its inverse function $\mathcal{G}_{-n}: \mathbb{T} \times \mathbb{T} \rightarrow R^{+}$is then given by

$$
\widehat{\mathcal{G}}_{-n}(t, s)= \begin{cases}(t-s)^{-n}, & \text { if }[s, t] \text { dense; } \\ \frac{1}{\prod_{j=0}^{n-1}\left(\rho^{n}(t)-\rho^{j}(s)\right)}, & \text { if }[s, t] \text { isolated }\end{cases}
$$

We use the convention $\widehat{\mathcal{G}_{0}}(t, s)=1$ for all $s, t \in \mathbb{T}$.

Notice that

$$
\widehat{\mathcal{G}}_{-n}\left(\sigma^{n}(t), s\right)=\frac{1}{\widehat{\mathcal{G}}_{n}(t, s)}, \quad s, t \in \mathbb{T}, s<t .
$$

Corollary 2.5 For $h>0, \mathbb{T}=h \mathbb{Z}=\{h k: k \in \mathbb{Z}\}$, we have $\rho^{k}(s)=s-k h$. Then

$$
\widehat{\mathcal{G}}_{n}(t, s)=(t-s)_{h}^{(n)}=\prod_{j=0}^{n-1}(t-s+j h)=h^{n}\left(\frac{t-s}{h}\right)^{(n)}, \quad n \in \mathbb{N},
$$

and

$$
\widehat{\mathcal{G}}_{-n}(t, s)=(t-s)_{h}^{(-n)}=\frac{1}{\prod_{j=0}^{n-1}(t-n-s+j h)}=h^{-n}\left(\frac{t-n-s}{h}+n\right)^{(-n)}, \quad n \in \mathbb{N},
$$

where

$$
x^{(n)}=\frac{\Gamma(x+n)}{\Gamma(x)} \quad \text { and } \quad x^{(-n)}=\frac{1}{(x-n)^{(n)}}=\frac{\Gamma(x-n)}{\Gamma(x)}, \quad n \in \mathbb{N}, x^{(0)}=1 .
$$

For $\mathbb{T}=q^{\mathbb{N}_{0}}$, we have $\rho^{k}(s)=s q^{-k}$. Then we write

$$
\widehat{\mathcal{G}}_{n}(t, s)=(t-s)_{\tilde{q}}^{(n)}=\prod_{j=0}^{n-1}\left(t-s q^{-j}\right)=t^{n} \prod_{j=0}^{n-1}\left(1-\frac{\tilde{q}^{j} s}{t}\right) \quad\left(0<\tilde{q}=\frac{1}{q}<1\right) .
$$

Remark 2.6 Regarding the generalization of the power function, $\widehat{\mathcal{G}}_{\alpha}(t, s)$ to real values of $\alpha \geq 0$ (instead of integers $n$ ), we recall broadly accepted extension of its particular cases (2.5) and (2.7) in the form (see [37])

$$
(t-s)_{h}^{(\alpha)}=h^{\alpha} \frac{\Gamma\left(\frac{t-s}{h}+\alpha\right)}{\Gamma\left(\frac{t-s}{h}\right)}, \quad(t-s)_{\tilde{q}}^{(\alpha)}=t^{\alpha} \frac{(s / t, \tilde{q})_{\infty}}{\left(\tilde{q}^{\alpha} s / t, \tilde{q}\right)_{\infty}}, \quad t \neq 0
$$

where $(p, \tilde{q})_{\infty}=\prod_{j=0}^{\infty}\left(1-p \tilde{q}^{j}\right)$. 
Remark 2.7 The integer iteration of the functions $\sigma^{n}, \rho^{n}$ follows the standard composition rule. The problem of noninteger iteration of the functions $\sigma^{\gamma}, \rho^{\gamma}$ for $\gamma \in \mathbb{R}$ on discrete time scales has been discussed in [37]. In particular, for $\mathbb{T}=h Z, h>0$, we have

$$
\sigma^{\gamma}(t)=t+\gamma h \quad \text { and } \quad \rho^{\gamma}(t)=t-\gamma h
$$

For $\mathbb{T}=\overline{q^{\mathbb{Z}}}, q>1$, we have

$$
\sigma^{\gamma}(t)=q^{\gamma} t+[\gamma]_{q} h \quad \text { and } \quad \rho^{\gamma}(t)=q^{-\gamma}\left(t-[\gamma]_{q} h\right)
$$

where $[\gamma]_{q}=\frac{q^{\gamma}-1}{q-1}$.

We also have

$$
(\sigma \circ \rho)(t)=(\rho \circ \sigma)(t)=t
$$

which implies $\sigma=\rho^{-1}$ and $\sigma^{-1}=\rho$, respectively.

\section{Conformable nabla derivative}

Several variants of conformable (fractional) derivative have been defined on time scales (see $[22,28,30])$. In this section we give the definition of conformable nabla derivative (strictly following [20]) depending on the function $\widehat{\mathcal{G}}_{1-\gamma}(t, a)$ (as defined in Sect. 2 above).

Throughout the paper, the operator $\nabla_{a}^{\gamma}$ is referred to as the conformable fractional nabla derivative of order $\gamma \in(0,1]$ on $\mathbb{T}$ at a point $t>a \in \mathbb{T}$.

Definition 3.1 Given a function $f: \mathbb{T} \rightarrow \mathbb{R}$ and $a \in \mathbb{T}, f$ is $(\gamma, a)$-nabla differentiable at $t>a$, if it is nabla differentiable at $t$, and its $(\gamma, a)$-nabla derivative is defined by

$$
\nabla_{a}^{\gamma} f(t)=\widehat{\mathcal{G}}_{1-\gamma}(t, a) f^{\nabla}(t), \quad t>a,
$$

where the function $\widehat{\mathcal{G}}_{1-\gamma}(t, a)$ as defined in (2.2).

If $\nabla_{a}^{\gamma}[f(t)]$ exists in some interval $(a, a+\epsilon)_{\mathbb{T}}, \epsilon>0$, then we define

$$
\nabla_{a}^{\gamma}[f(a)]=\lim _{t \rightarrow a^{+}} \nabla_{a}^{\gamma}[f(t)]
$$

if the limit $\lim _{t \rightarrow a^{+}} \nabla_{a}^{\gamma}[f(t)]$ exists.

Moreover, we call $f$ is $(\gamma, a)$-nabla differentiable on $\mathbb{T}_{\kappa}\left(a \in \mathbb{T}_{\kappa}\right)$ provided $\nabla_{a}^{\gamma}[f(t)]$ exists for all $t \in \mathbb{T}_{\kappa}$. The function $\nabla_{a}^{\gamma}: \mathbb{T}_{\kappa} \rightarrow \mathbb{R}$ is then called the $(\gamma, a)$-nabla derivative of $f$ on $\mathbb{T}_{\kappa}$.

Some useful properties concerning the $(\gamma, a)$-nabla derivative are given next. The proof of this theorem is similar to that in [23], hence omitted.

Theorem 3.2 If $: \mathbb{T} \rightarrow \mathbb{R}$ is $(\gamma, a)$-nabla differentiable at $t \in \mathbb{T}_{\kappa}$, where $t>a$ and $\gamma \in$ $(0,1]$, then $f$ is continuous at $t$. 
Remark 3.3 For $\mathbb{T}=\mathbb{R}$ and $t>a \in \mathbb{R}$, then we have

$$
\nabla_{a}^{\gamma}[f(t)]=f^{\prime}(t)(t-a)^{1-\gamma}
$$

which is the conformable fractional derivative defined in [5].

One the other hand, if $t \in \mathbb{Z}$ and $t>a$, then we have the $(\gamma, a)$-(backward) difference

$$
\nabla_{a}^{\gamma}[f(t)]:=[f(t)-f(t-1)](t-a)^{(1-\gamma)},
$$

a new definition in discrete setting.

\section{Example 3.4}

(i) Let $a \in \mathbb{T}$. If $f: \mathbb{T} \rightarrow \mathbb{R}$ is defined by $f(t)=\alpha$ for all $t \in \mathbb{T}, t>a$, where $\alpha \in \mathbb{R}$ a constant, then we have $\nabla_{a}^{\gamma}[\alpha]=0$.

(ii) Let $a \in \mathbb{T}$. If $f: \mathbb{T} \rightarrow \mathbb{R}$ is defined by $f(t)=t-a$ for all $t \in \mathbb{T}, t>a$, then

$$
\nabla_{a}^{\gamma}[t-a]=\widehat{\mathcal{G}}_{1-\gamma}(t, a) \quad \text { for all } t \in \mathbb{T}
$$

(iii) If $f: \mathbb{T} \rightarrow \mathbb{R}$ is defined by $f(t)=t^{2}$ for all $t \in[a, b]_{\mathbb{T}}$, then

$$
\nabla_{a}^{\gamma}\left[t^{2}\right]=(\rho(t)+t) \widehat{\mathcal{G}}_{1-\gamma}(t, a) \quad \text { for all } t \in[a, b]_{\mathbb{T}}
$$

Next, we provide the $(\gamma, a)$-nabla derivatives of sums, products, and quotients of $(\gamma, a)$ nabla differentiable functions. The proof is omitted since it is similar to that in [23].

Theorem 3.5 Assume $f, g: \mathbb{T} \rightarrow \mathbb{R}$ are $(\gamma, a)$-nabla differentiable at $t \in \mathbb{T}_{\kappa}, t>a$. Then:

(i) The $\operatorname{sum} f+g: \mathbb{T} \rightarrow \mathbb{R}$ is $(\gamma, a)$-nabla differentiable at $t$ with

$$
\nabla_{a}^{\gamma}(r f+s g)(t)=r \nabla_{a}^{\gamma} f(t)+s \nabla_{a}^{\gamma} g(t)
$$

(ii) For any $k \in \mathbb{R}, k f: \mathbb{T} \rightarrow \mathbb{R}$ is $(\gamma, a)$-nabla differentiable at $t$ with

$$
\nabla_{a}^{\gamma}(k f)(t)=k \nabla_{a}^{\gamma} f(t)
$$

(iii) The product $f g: \mathbb{T} \rightarrow \mathbb{R}$ is $(\gamma, a)$-nabla differentiable at $t$ with

$$
\nabla_{a}^{\gamma}(f g)(t)=\left[\nabla_{a}^{\gamma} f(t)\right] g(t)+f(\rho(t)) \nabla_{a}^{\gamma} g(t)=f(t) \nabla_{a}^{\gamma} g(t)+\nabla_{a}^{\gamma} f(t)[g(\rho(t))] .
$$

(iv) If $g(t) g(\rho(t)) \neq 0$, then $\frac{f}{g}$ is $(\gamma, a)$-nabla differentiable at $t$ with

$$
\nabla_{a}^{\gamma}\left(\frac{f}{g}\right)(t)=-\frac{\left[\nabla_{a}^{\gamma} f(t)\right] g(t)-f(t) \nabla_{a}^{\gamma} g(t)}{g(t) g(\rho(t))}
$$

In the case $f \equiv 1$, we have

$$
\nabla_{a}^{\gamma}\left(\frac{1}{g}\right)(t)=-\frac{\nabla_{a}^{\gamma} g(t)}{g(t) g(\rho(t))}
$$


Lemma 3.6 (Chain rule) Let $g \in C_{\mathrm{ld}}^{\nabla}(\mathbb{T})$ and assume that $f: \mathbb{R} \rightarrow \mathbb{R}$ is continuously differentiable. Then $f \circ g: \mathbb{T} \rightarrow \mathbb{R}$ is $(\gamma, a)$-nabla differentiable and satisfies

$$
\nabla_{a}^{\gamma}[(f \circ g)(t)]=\left\{\int_{0}^{1} f^{\prime}\left(g(t)-h v(t) g^{\nabla}(t)\right) d h\right\} \nabla_{a}^{\gamma} g(t)
$$

Proof The nabla version of the chain rule is given by

$$
(f \circ g)^{\nabla}(t)=\left\{\int_{0}^{1} f^{\prime}\left(g(t)-h v(t) g^{\nabla}(t)\right) d h\right\} g^{\nabla}(t) .
$$

This can be obtained by following the proof of the chain rule in [34] and applying the ordinary substitution rule from calculus to find

$$
\begin{aligned}
f(g(\rho(t)))-f(g(s)) & =\int_{g(s)}^{g(\rho(t))} f^{\prime}(\tau) d \tau \\
& =[g(\rho(t))-g(s)] \int_{0}^{1} f^{\prime}[h g(\rho(t))+(1-h) g(s)] d h .
\end{aligned}
$$

Hence, we have

$$
\begin{aligned}
\nabla_{a}^{\gamma}[(f \circ g)(t)] & =\widehat{\mathcal{G}_{1-\gamma}}(t, a)(f \circ g)^{\nabla}(t) \\
& =\widehat{\mathcal{G}_{1-\gamma}}(t, a)\left\{\int_{0}^{1} f^{\prime}\left(g(t)-h v(t) g^{\nabla}(t)\right) d h\right\} g^{\nabla}(t) \\
& =\left\{\int_{0}^{1} f^{\prime}\left(g(t)-h v(t) g^{\nabla}(t)\right) d h\right\} \nabla_{a}^{\gamma} g(t) .
\end{aligned}
$$

This completes the proof.

Lemma 3.7 Let $m \in \mathbb{N}, a \in \mathbb{T}$ and suppose $f: \mathbb{T} \rightarrow \mathbb{R}$ is $(\gamma, a)$-nabla differentiableat $t \in \mathbb{T}$.

(i) For $f$ defined by $f(t)=\widehat{\mathcal{G}}_{m}(t, s), s \in \mathbb{T}$, we have

$$
\nabla_{a}^{\gamma} f(t)=\widehat{\mathcal{G}}_{1-\gamma}(t, a) \sum_{k=0}^{m-1} \widehat{\mathcal{G}}_{k}(\rho(t), s) \widehat{\mathcal{G}}_{m-1-k}(t, s) .
$$

(ii) For $g$ defined by $g(t)=\widehat{\mathcal{G}}_{-m}\left(\sigma^{m}(t), s\right), s \in \mathbb{T}$, we have

$$
\nabla_{a}^{\gamma} g(t)=-\widehat{\mathcal{G}}_{1-\gamma}(t, a) \sum_{k=0}^{m-1} \frac{1}{\widehat{\mathcal{G}}_{m-k}\left(\sigma^{k-1}(t), s\right) \widehat{\mathcal{G}}_{k+1}\left(\sigma^{m-1-k}(t), s\right)} .
$$

Proof It is sufficient to prove the results for a discrete time scale $\mathbb{T}$. We will prove by induction. If $m=1$, then $f(t)=\widehat{\mathcal{G}_{1}}(t, s)=t-s$ and, clearly, $\nabla_{a}^{\gamma}(t)=\widehat{\mathcal{G}_{1-\gamma}}(t, a)$ holds by Example 3.4(ii). Now we assume that

$$
\nabla_{a}^{\gamma} f(t)=\widehat{\mathcal{G}}_{1-\gamma}(t, a) \sum_{k=0}^{m-1} \widehat{\mathcal{G}}_{k}(\rho(t), s) \widehat{\mathcal{G}}_{m-1-k}(t, s)
$$


holds for $f(t)=\widehat{\mathcal{G}}_{m}(t, s)$. Let

$$
F(t)=\widehat{\mathcal{G}}_{m+1}(t, s)=\prod_{j=0}^{m}\left(t-\rho^{j}(s)\right)=\left(t-\rho^{m}(s)\right) \prod_{j=0}^{m-1}\left(t-\rho^{j}(s)\right)=\left(t-\rho^{m}(s)\right) f(t) .
$$

By the product rule (Theorem 3.5(iii)), we obtain

$$
\begin{aligned}
\nabla_{a}^{\gamma} F(t) & =\widehat{\mathcal{G}}_{1-\gamma}(t, a) f(\rho(t))+\left(t-\rho^{m}(s)\right) \nabla_{a}^{\gamma}(t) \\
& =\widehat{\mathcal{G}}_{1-\gamma}(t, a) \widehat{\mathcal{G}}_{m}(\rho(t), s)+\left(t-\rho^{m}(s)\right) \widehat{\mathcal{G}}_{1-\gamma}(t, a) \sum_{k=0}^{m-1} \widehat{\mathcal{G}}_{k}(\rho(t), s) \widehat{\mathcal{G}}_{m-1-k}(t, s) \\
& =\widehat{\mathcal{G}}_{1-\gamma}(t, a)\left[\widehat{\mathcal{G}}_{m}(\rho(t), s)+\left(t-\rho^{m}(s)\right) \sum_{k=0}^{m-1} \widehat{\mathcal{G}}_{k}(\rho(t), s) \widehat{\mathcal{G}}_{m-1-k}(t, s)\right] \\
& =\widehat{\mathcal{G}}_{1-\gamma}(t, a)\left[\widehat{\mathcal{G}}_{m}(\rho(t), s)+\sum_{k=0}^{m-1} \widehat{\mathcal{G}}_{k}(\rho(t), s) \widehat{\mathcal{G}}_{m-k}(t, s)\right] \\
& =\widehat{\mathcal{G}}_{1-\gamma}(t, a) \sum_{k=0}^{m} \widehat{\mathcal{G}}_{k}(\rho(t), s) \widehat{\mathcal{G}}_{m-k}(t, s) .
\end{aligned}
$$

Hence, by mathematical induction, part (i) holds.

Next, for $g(t)=\widehat{\mathcal{G}}_{-m}\left(\sigma^{m}(t), s\right)=\frac{1}{f(t)}$ we apply Theorem 3.5(iv) to obtain

$$
\begin{aligned}
\left(\nabla_{a}^{\gamma} g\right)(t) & =-\frac{\nabla_{a}^{\gamma}(t)}{f(t) f(\rho(t))} \\
& =-\frac{\widehat{\mathcal{G}_{1-\gamma}}(t, a) \sum_{k=0}^{m-1} \widehat{\mathcal{G}}_{k}(\rho(t), s) \widehat{\mathcal{G}}_{m-1-k}(t, s)}{\widehat{\mathcal{G}}_{m}(t, s) \widehat{\mathcal{G}}_{m}(\rho(t), s)} \\
& =-\widehat{\mathcal{G}}_{1-\gamma}(t, a) \sum_{k=0}^{m-1} \frac{\widehat{\mathcal{G}}_{k}(\rho(t), s) \widehat{\mathcal{G}}_{m-1-k}(t, s)}{\widehat{\mathcal{G}}_{m}(t, s) \widehat{\mathcal{G}}_{m}(\rho(t), s)} \\
& =-\widehat{\mathcal{G}}_{1-\gamma}(t, a) \sum_{k=0}^{m-1} \frac{\prod_{j=0}^{k-1}\left(\rho(t)-\rho^{j}(s)\right) \prod_{j=0}^{m-2-k}\left(t-\rho^{j}(s)\right)}{\prod_{j=0}^{m-1}\left(\rho(t)-\rho^{j}(s)\right) \prod_{j=0}^{m-1}\left(t-\rho^{j}(s)\right)} \\
& =-\widehat{\mathcal{G}}_{1-\gamma}(t, a) \sum_{k=0}^{m-1} \frac{1}{\prod_{j=k}^{m-1}\left(\rho(t)-\rho^{j}(s)\right) \prod_{j=m-1-k}^{m-1}\left(t-\rho^{j}(s)\right)} \\
& =-\widehat{\mathcal{G}}_{1-\gamma}(t, a) \sum_{k=0}^{m-1} \frac{1}{\prod_{j=0}^{m-1-k}\left(\sigma^{k-1}(t)-\rho^{j}(s)\right) \prod_{j=0}^{k}\left(\sigma^{m-1-k}(t)-\rho^{j}(s)\right)} \\
& =-\widehat{\mathcal{G}}_{1-\gamma}(t, a) \sum_{k=0}^{m-1} \frac{1}{\widehat{\mathcal{G}}_{m-k}\left(\sigma^{k-1}(t), s\right) \widehat{\mathcal{G}}_{k+1}\left(\sigma^{m-1-k}(t), s\right)},
\end{aligned}
$$

provided $\widehat{\mathcal{G}}_{m-k}(\sigma(t), s) \widehat{\mathcal{G}}_{k+1}(\sigma(t), s) \neq 0$.

Example 3.8 Consider the time scale

$$
\mathbb{T}=\mathbb{N}_{0}^{\frac{1}{2}}:=\left\{\sqrt{n}: n \in \mathbb{N}_{0}\right\} .
$$


We have $\sigma(t)=\sqrt{t^{2}+1}$ and $\rho(t)=\sqrt{t^{2}-1}$ for $t \in \mathbb{T}$. Let $\gamma=\frac{1}{2}, a=s=0 \in \mathbb{T}$.

(i) Let $f(t)=\widehat{\mathcal{G}_{2}}(t, 0)=t^{(2)}$ for $t \in \mathbb{T}(t>1)$, then we have

$$
\begin{aligned}
\nabla_{0}^{\frac{1}{2}}\left[t^{(2)}\right] & =t^{\left(\frac{1}{2}\right)} \sum_{k=0}^{1} \widehat{\mathcal{G}}_{k}\left(\sqrt{t^{2}-1}, 0\right) \widehat{\mathcal{G}}_{1-k}(t, 0) \\
& =t^{\left(\frac{1}{2}\right)}\left[t^{(1)}+\left(\sqrt{t^{2}-1}\right)^{(1)}\right] \\
& =\left[t+\sqrt{t^{2}-1}\right] t^{\left(\frac{1}{2}\right)} .
\end{aligned}
$$

(ii) Let $g(t)=\widehat{\mathcal{G}}_{-2}\left(\sigma^{2}(t), 0\right)=\frac{1}{t^{(2)}}$ for $t \in \mathbb{T}(t>1)$, then

$$
\begin{aligned}
\nabla_{0}^{\frac{1}{2}}\left[\frac{1}{t^{(2)}}\right] & =-t^{\left(\frac{1}{2}\right)} \sum_{k=0}^{1} \frac{1}{\widehat{\mathcal{G}}_{2-k}\left(\sqrt{t^{2}+k-1}, 0\right) \widehat{\mathcal{G}}_{k+1}\left(\sqrt{t^{2}+1-k}, 0\right)} \\
& =-\left[\frac{1}{\left(\sqrt{t^{2}-1}\right)^{(2)}\left(\sqrt{t^{2}+1}\right)}+\frac{1}{t^{(2)}(t)}\right] t^{\left(\frac{1}{2}\right)} .
\end{aligned}
$$

\section{Conformable $\nabla$-integrals}

In this section, we give the definition of conformable fractional $\nabla$-integral of order $\gamma \in$ $(0,1]$ starting from $a \in \mathbb{T}$ (or simply $\gamma$-nabla integral from $a$ ) on a time scale $\mathbb{T}$.

Definition 4.1 Assume that $\gamma \in(0,1], a, t_{1}, t_{2} \in \mathbb{T}, a \leq t_{1} \leq t_{2}$ and $f \in C_{\mathrm{ld}}(\mathbb{T})$, then we say that $f$ is $(\gamma, a)$-nabla integrable on the interval $\left[t_{1}, t_{2}\right]_{\mathbb{T}}$ if the following integral:

$$
\nabla_{a}^{-\gamma} f(t)=\int_{t_{1}}^{t_{2}} f(\tau) \nabla_{a}^{\gamma} \tau=\int_{t_{1}}^{t_{2}} f(\tau) \widehat{\mathcal{G}}_{\gamma-1}\left(\sigma^{\gamma-1}(\tau), a\right) \nabla \tau
$$

exists and is finite.

Remark 4.2 If $t_{1} \in \mathbb{T}$ and $\sup \mathbb{T}=\infty$, and $f \in C_{\text {ld }}(\mathbb{T})$, then the improper nabla integral has the form

$$
\int_{t_{1}}^{\infty} f(\tau) \nabla_{a}^{\gamma} \tau=\lim _{t \rightarrow \infty} \int_{t_{1}}^{t} f(\tau) \nabla_{a}^{\gamma} \tau
$$

Example 4.3 For the case $\mathbb{T}=\mathbb{R}$, we have the classical conformable integral as defined in [5], namely

$$
\int_{a}^{t} f(\tau) \nabla_{a}^{\gamma} \tau=\int_{a}^{t} f(\tau)(\tau-a)^{\gamma-1} d \tau .
$$

For $\mathbb{T}=h \mathbb{Z} h>0$, we have a new conformable fractional $h$-sum given by

$$
\int_{a}^{t} f(\tau) \nabla_{a}^{\gamma} \tau=\sum_{\tau \in(a, t]} h f(\tau)\left(\rho^{\gamma-1}(\tau)-a\right)_{h}^{(\gamma-1)}=h^{\gamma} \sum_{\tau \in(a, t]} f(\tau) \frac{\Gamma\left(\frac{\tau-a}{h}\right)}{\Gamma\left(\frac{\tau-a}{h}-\gamma+1\right)} .
$$

For $\mathbb{T}=q^{\mathbb{N}_{0}}$, we have a new conformable fractional $q$-sum given by

$$
\int_{a}^{t} f(\tau) \nabla_{a}^{\gamma} \tau=\sum_{\tau \in(a, t]} \tau(1-\tilde{q}) f(\tau)\left(\rho^{\gamma-1}(\tau)-a\right)_{\tilde{q}}^{(\gamma-1)}=(1-\tilde{q}) \sum_{\tau \in(a, t]} \tau f(\tau)\left(\tilde{q}^{\gamma-1} \tau-a\right)_{\tilde{q}}^{(\gamma-1)} .
$$


Example 4.4 Let $\mathbb{T}=\mathbb{Z}, a=0$ and $\gamma=1 / 2$. Then

$$
\begin{aligned}
\int_{0}^{3} 3^{t} \nabla_{0}^{\frac{1}{2}} t & =\sum_{t=1}^{3} 3^{t}(t-1 / 2)^{(-1 / 2)}=\sum_{t=1}^{3} 3^{t} \frac{\Gamma(t)}{\Gamma(t-1 / 2)} \\
& =\frac{\Gamma(1)}{\Gamma(1 / 2)}+(9) \frac{\Gamma(2)}{\Gamma(3 / 2)}+(27) \frac{\Gamma(3)}{\Gamma(5 / 2)} \\
& =\frac{1}{\sqrt{2}}+\frac{18}{\sqrt{2}}+\frac{72}{\sqrt{2}} \\
& =\frac{91}{\sqrt{2}} .
\end{aligned}
$$

The proof of the following theorem is similar to that of Theorem 8 in [23], hence omitted.

Theorem 4.5 Let $\gamma \in(0,1]$ and $a \in \mathbb{T}$. Then, for any ld-continuous function $f: \mathbb{T} \rightarrow \mathbb{R}$, there exists a function $F: \mathbb{T} \rightarrow \mathbb{R}$ such that

$$
\nabla_{a}^{\gamma} F(t)=f(t) \quad \text { for all } t \in \mathbb{T}_{\kappa} .
$$

The function $F$ is called an $(\gamma, a)$-nabla antiderivative of $f$.

Lemma 4.6 Assume $a, t_{1}, t_{2} \in \mathbb{T}, a \leq t_{1} \leq t_{2}$ and $f \in C_{\mathrm{ld}}(\mathbb{T})$. Let the function $F: \mathbb{T} \rightarrow \mathbb{R}$ be $(\gamma, a)$-nabla differentiable on $\left[t_{1}, t_{2}\right]$ such that

$$
\nabla_{a}^{\gamma} F(t)=f(t) \quad \text { holds for all } t \in\left[t_{1}, t_{2}\right]_{\mathbb{T}} \text {. }
$$

Then

$$
\int_{t_{1}}^{t_{2}} f(\tau) \nabla_{a}^{\gamma} \tau=F\left(t_{2}\right)-F\left(t_{1}\right)
$$

Proof Since $f \in C_{\mathrm{ld}}(\mathbb{T})$, by Theorem 4.5 , we have

$$
\int_{t_{1}}^{t_{2}} f(\tau) \nabla_{a}^{\gamma} \tau=\int_{t_{1}}^{t_{2}} \nabla_{a}^{\gamma} F(t) \nabla_{a}^{\gamma} \tau=\int_{t_{1}}^{t_{2}} F^{\nabla}(\tau) \nabla \tau=F\left(t_{1}\right)-F\left(t_{2}\right)
$$

Similar to the proof of Theorem 9 in [23] (see also [34]), one can prove the following theorem.

Theorem 4.7 Let $\gamma \in(0,1]$. If $a, t_{1}, t_{2}, t_{3} \in \mathbb{T}, a \leq t_{1} \leq t_{2} \leq t_{3}, \alpha \in \mathbb{R}$, and $f, g \in C_{\mathrm{ld}}(\mathbb{T})$, then

(i) $\int_{t_{1}}^{t_{2}}[f(t)+g(t)] \nabla_{a}^{\gamma} t=\int_{t_{1}}^{t_{2}} f(t) \nabla_{a}^{\gamma} t+\int_{t_{1}}^{t_{2}} g(t) \nabla_{a}^{\gamma} t$;

(ii) $\int_{t_{1}}^{t_{2}} \alpha f(t) \nabla_{a}^{\gamma} t=\alpha \int_{t_{1}}^{t_{2}} f(t) \nabla_{a}^{\gamma} t$;

(iii) $\int_{t_{1}}^{t_{2}} f(t) \nabla_{a}^{\gamma} t=-\int_{t_{2}}^{t_{1}} f(t) \nabla_{a}^{\gamma} t$;

(iii) $\int_{t_{1}}^{t_{3}} f(t) \nabla_{a}^{\gamma} t=\int_{t_{1}}^{t_{2}} f(t) \nabla_{a}^{\gamma} t+\int_{t_{2}}^{t_{3}} f(t) \nabla_{a}^{\gamma} t$;

(iv) $\int_{t_{1}}^{t_{2}}\left[f(t)\left(\nabla_{a}^{\gamma} g\right)(t)\right] \nabla_{a}^{\gamma} t=[f(t) g(t)]_{t_{1}}^{t_{2}}-\int_{t_{1}}^{t_{2}} \nabla_{a}^{\gamma}(t) g(\rho(t)) \nabla_{a}^{\gamma} t$;

(v) $\int_{t_{1}}^{t_{1}} f(t) \nabla_{a}^{\gamma} t=0$; 
(vi) If $|f(t)| \leq g(t)$ on $\left[t_{1}, t_{2}\right)$, then

$$
\left|\int_{t_{1}}^{t_{2}} f(t) \nabla_{a}^{\gamma} t\right| \leq \int_{t_{1}}^{t_{2}} g(t) \nabla_{a}^{\gamma} t
$$

Theorem 4.8 Assume $\gamma \in(0,1]$ and $a \in \mathbb{T}$. If $\in C_{\mathrm{ld}}(\mathbb{T})$ and $t \in \mathbb{T}_{\kappa}$ with $\rho(t)>a$, then

$$
\int_{\rho(t)}^{t} f(\tau) \nabla_{a}^{\gamma} \tau=v(t) f(t) \widehat{\mathcal{G}}_{\gamma-1}\left(\sigma^{\gamma-1}(t), a\right) .
$$

Proof Since $f \in C_{\mathrm{ld}}(\mathbb{T})$, by Lemma 4.6, we have

$$
\begin{aligned}
\int_{\rho(t)}^{t} f(\tau) \nabla_{a}^{\gamma} \tau & =F(t)-F(\rho(t)) \\
& =v(t)\left[\frac{F(t)-F(\rho(t))}{v(t)}\right] \frac{\widehat{\mathcal{G}}_{1-\gamma}(t, a)}{\widehat{\mathcal{G}}_{1-\gamma}(t, a)} \\
& =v(t)\left[F^{\nabla}(t) \widehat{\mathcal{G}}_{1-\gamma}(t, a)\right] \widehat{\mathcal{G}}_{\gamma-1}\left(\sigma^{\gamma-1}(\tau), a\right) \\
& =v(t) f(t) \widehat{\mathcal{G}}_{\gamma-1}\left(\sigma^{\gamma-1}(\tau), a\right) .
\end{aligned}
$$

Theorem 4.9 Let $\gamma \in(0,1], a, t \in \mathbb{T}, t \geq a$ and $f \in C_{\mathrm{ld}}(\mathbb{T})$. Then, for all $t \in \mathbb{T}$, we have

$$
\nabla_{a}^{\gamma}\left(\nabla_{a}^{-\gamma} f\right)(t)=f(t)
$$

Proof Since $f \in C_{\mathrm{ld}}(\mathbb{T})$ and $\nabla_{a}^{-\gamma} f(t)$ is $(\gamma, a)$-nabla integrable, we have

$$
\begin{aligned}
\nabla_{a}^{\gamma}\left(\nabla_{a}^{-\gamma} f\right)(t) & =\widehat{\mathcal{G}}_{1-\gamma}(t, a)\left[\nabla_{a}^{-\gamma}(f)(t)\right]^{\nabla} \\
& =\widehat{\mathcal{G}}_{1-\gamma}(t, a)\left[\int_{a}^{t} f(\tau) \widehat{\mathcal{G}}_{\gamma-1}\left(\sigma^{\gamma-1}(\tau), a\right) \nabla \tau\right]^{\nabla} \\
& =\widehat{\mathcal{G}}_{1-\gamma}(t, a)\left[f(t) \frac{1}{\widehat{\mathcal{G}}_{1-\gamma}(t, a)}\right] \\
& =f(t) .
\end{aligned}
$$

Theorem 4.10 Let $f:[a, b]_{\mathbb{T}} \rightarrow \mathbb{R}$ be $(\eta, a)$-differentiable for $\eta \in(0,1]$. Then, for all $t>a$, we have

$$
\nabla_{a}^{-\gamma} \nabla_{a}^{\gamma} f(t)=f(t)-f(a)
$$

Proof Since $f$ is differentiable, by Definition 4.1, we have

$$
\begin{aligned}
\nabla_{a}^{-\gamma} \nabla_{a}^{\gamma} f(t) & =\int_{a}^{t} \nabla_{a}^{\gamma} f(\tau) \widehat{\mathcal{G}}_{\gamma-1}\left(\sigma^{\gamma-1}(\tau), a\right) \nabla \tau \\
& =\int_{a}^{t} f^{\nabla}(t) \widehat{\mathcal{G}}_{1-\gamma}(\tau, a) \frac{1}{\widehat{\mathcal{G}}_{1-\gamma}(\tau, a)} \nabla \tau \\
& =\int_{a}^{t} f^{\nabla}(\tau) \nabla \tau \\
& =f(t)-f(a) .
\end{aligned}
$$




\section{Conformable exponential function}

In this section we define the conformable fractional nabla exponential function on time scales.

Definition 5.1 The function $p: \mathbb{T} \rightarrow \mathbb{R}$ is said to be $v$-regressive if

$$
1-v(t) p(t) \neq 0 \quad \text { for all } t \in \mathbb{T}_{\kappa}
$$

holds. Define the $v$-regressive class of ld-functions on $\mathbb{T}_{\kappa}$ to be

$$
\mathcal{R}_{v}:=\left\{p \in C_{\mathrm{ld}}(\mathbb{T}): 1-v(t) p(t) \neq 0\right\}
$$

and define the set $\mathcal{R}_{v}^{+}$of all positive regressive elements of $\mathcal{R}_{v}$ by

$$
\mathcal{R}_{v}^{+}=\left\{p \in \mathcal{R}_{v}: 1-v(t) p(t)>0 \text { for all } t \in \mathbb{T}\right\} .
$$

Definition 5.2 For $p \in \mathcal{R}_{v}$, define circle minus $p$ by

$$
\ominus_{\nu} p:=-\frac{p}{1-p \nu} .
$$

Definition 5.3 For $h>0$, let

$$
\mathbb{Z}_{h}:=\left\{z \in \mathbb{C}:-\frac{\pi}{h}<\operatorname{Im}(z)<\frac{\pi}{h}\right\} \quad \text { and } \quad \mathbb{C}_{h}:=\left\{z \in \mathbb{C}: z \neq \frac{1}{h}\right\} .
$$

Define the $v$-cylinder transformation $\widehat{\xi}_{h}(z): \mathbb{C}_{h} \rightarrow \mathbb{Z}_{h}$ by

$$
\widehat{\xi}_{h}(z):= \begin{cases}-\frac{1}{h} \log (1-z h), & h \neq 0 ; \\ z, & h=0,\end{cases}
$$

where $\log$ is the principle logarithm function.

The inverse transform $\widehat{\xi}_{h}^{-1}$ is then given by

$$
\widehat{\xi}_{h}^{-1}(z)= \begin{cases}\frac{1}{h}\left(1-e^{-z h}\right), & h \neq 0 \\ z, & h=0 .\end{cases}
$$

The following lemma is an easy exercise.

\section{Lemma 5.4 It holds that}

$$
\widehat{\xi}_{h}\left(\ominus_{v} p\right)=-\widehat{\xi}_{h}(p) \text { for all } p \in \mathcal{R}_{v} .
$$

Definition 5.5 Let $\alpha \in(0,1], a, s, t \in \mathbb{T}, a \leq s \leq t$ and $p \in \mathcal{R}_{v}$. Then we define the (conformable) fractional nabla exponential function of order $\gamma$ from $a$ by

$$
\widehat{e}_{p}^{\gamma}(t, s ; a)=\exp \left(\int_{s}^{t} \widehat{\xi}_{v(\tau)}(p(\tau)) \nabla_{a}^{\gamma} \tau\right) \quad \text { for } a, t \in \mathbb{T} \text {. }
$$


Remark 5.6 In the case $s=a$, we write $\widehat{e}_{p}^{\gamma}(t, a ; a)=\widehat{e}_{p}^{\gamma}(t, a)$ and, if $\gamma=1$, we have the usual nabla exponential function as defined in [35].

Example 5.7 Let $\mathbb{T}=\mathbb{R}$ and $\lambda \in \mathcal{R}_{v}$ a constant. Then

$$
\widehat{e}_{\lambda}^{\gamma}(t, a)=\exp \left(\int_{a}^{t} \lambda(\tau-a)^{\gamma-1} d \tau\right)=e^{\lambda \frac{(t-a)^{\gamma}}{\gamma}}
$$

Notice that, if $\gamma=1$, we have $\widehat{e}_{\lambda}^{\gamma}(t, a)=\widehat{e}_{\lambda}(t, a)=e^{\lambda(t-a)}$, which coincides with the classical exponential function.

Example 5.8 Let $t, a \in \mathbb{T}=\mathbb{Z}$ and let $\lambda \in \mathcal{R}_{v}$ be a constant. Then

$$
\widehat{e}_{\lambda}^{\gamma}(t, a)=\left(\frac{1}{1-\lambda}\right)^{\frac{(t-\gamma+1-a)(\gamma)}{\gamma}}, \quad t>a .
$$

Indeed, by (5.1) and (5.3), we have

$$
\begin{aligned}
\widehat{e}_{\lambda}^{\gamma}(t, a) & =\exp \left(-\int_{a}^{t} \log (1-\lambda)(\tau-\gamma+1-a)^{(\gamma-1)} \nabla \tau\right) \\
& =\exp \left(-\log (1-\lambda) \int_{a}^{t}(\tau-\gamma+1-a)^{(\gamma-1)} \nabla \tau\right),
\end{aligned}
$$

Since $\nabla t^{(\gamma)}=\gamma t^{(\gamma-1)}$, we obtain

$$
\int_{a}^{t}(\tau-\gamma+1-a)^{(\gamma-1)} \nabla \tau=\sum_{\tau=a+1}^{t}(\tau-\gamma+1-a)^{(\gamma-1)}=\frac{(t-\gamma+1-a)^{(\gamma)}}{\gamma} .
$$

It follows that

$$
\widehat{e}_{\lambda}^{\gamma}(t, a)=e^{-\frac{(t-\gamma+1-a)(\gamma)}{\gamma} \log (1-\lambda)}=\left(\frac{1}{1-\lambda}\right)^{\frac{(t-\gamma+1-a)(\gamma)}{\gamma}} .
$$

Note that, for $\gamma=1$, we have

$$
\widehat{e}_{\lambda}(t, a)=\left(\frac{1}{1-\lambda}\right)^{t-a}
$$

which coincides with the nabla exponential function given in [35].

Theorem 5.9 Let $p, q \in \mathcal{R}_{v}$ and $a, s, t \in \mathbb{T}(a<s \leq t)$. Then:

(i) $\widehat{e}_{0}^{\gamma}(t, a)=1, \widehat{e}_{p}^{\gamma}(a, a)=1$;

(ii) $\widehat{e}_{p}^{\gamma}(\rho(t), a)=(1-v(t) p(t))^{\widehat{\mathcal{G}}_{\gamma-1}\left(\sigma^{\gamma-1}(t), a\right)} \widehat{e}_{p}^{\gamma}(t, a)$;

(iii) $\frac{1}{\hat{e}_{p}^{\gamma}(t, a)}=\widehat{e}_{\ominus_{v} p}^{\gamma}(t, a)$;

(iv) $\widehat{e}_{p}^{\gamma}(t, a)=\frac{1}{\hat{e}_{p}^{\gamma}(a, t)}=\widehat{e}_{\ominus_{v} p}^{\gamma}(a, t)$;

(v) $\widehat{e}_{p}^{\gamma}(t, a) \widehat{e}_{\ominus_{\nu} p}^{\gamma}(s, a)=\widehat{e}_{p}^{\gamma}(t, s ; a)$;

(vi) $\widehat{e}_{p}^{\gamma}(t, a) \widehat{e}_{q}^{\gamma}(t, a)=\widehat{e}_{p \oplus_{\nu} q}^{\gamma}(t, a)$;

(vii) $\frac{\widehat{e}_{p}^{\gamma}(t, a)}{\widehat{e}_{q}^{\gamma}(t, a)}=\widehat{e}_{p \ominus_{v} q}^{\gamma}(t, a)$. 
Proof (i) Clear by definition.

(ii) It is sufficient to prove the property for left-scattered points (i.e., $\rho(t)<t$,

$$
\widehat{e}_{p}^{\gamma}(\rho(t), a)=\exp \left(\int_{a}^{t} \widehat{\xi}_{v(\tau)}(p(\tau)) \nabla_{a}^{\gamma} \tau\right) \exp \left(-\int_{\rho(t)}^{t} \widehat{\xi}_{v(\tau)}(p(\tau)) \nabla_{a}^{\gamma} \tau\right)
$$

By Theorem 4.8,

$$
\int_{\rho(t)}^{t} \widehat{\xi}_{v(\tau)}(p(\tau)) \nabla_{a}^{\gamma} \tau=v(t) \widehat{\xi}_{v(t)}(p(t)) \widehat{\mathcal{G}}_{\gamma-1}\left(\sigma^{\gamma-1}(t), a\right)=-\log (1-v(t) p(t))^{\widehat{\mathcal{G}}_{\gamma-1}\left(\sigma^{\gamma-1}(t), a\right)}
$$

and substituting into (5.4), we get the required property.

(iii) Follows directly from Lemma 5.4. Indeed,

$$
\frac{1}{\widehat{e}_{p}^{\gamma}(t, a)}=\exp \left(-\int_{a}^{t} \widehat{\xi}_{v(\tau)}(p(\tau)) \nabla_{a}^{\gamma} \tau\right)=\exp \left(\int_{a}^{t} \widehat{\xi}_{v(\tau)}\left(\ominus_{\nu} p(\tau)\right) \nabla_{a}^{\gamma} \tau\right)
$$

hence the property (iii).

(iv) The result follows directly from (5.3) and part (iii) of this theorem.

(v) By Lemma 5.4 and Definition 5.5, we have

$$
\begin{aligned}
\widehat{e}_{p}^{\gamma}(t, a) \widehat{e}_{\ominus_{v} p}^{\gamma}(s, a) & =\exp \left(\int_{a}^{t} \widehat{\xi}_{v(\tau)}(p(\tau)) \nabla_{a}^{\gamma} \tau-\int_{a}^{s} \widehat{\xi}_{v(\tau)}(p(\tau)) \nabla_{a}^{\gamma} \tau\right) \\
& =\exp \left(\int_{a}^{t} \widehat{\xi}_{v(\tau)}(p(\tau)) \nabla_{a}^{\gamma} \tau+\int_{s}^{a} \widehat{\xi}_{v(\tau)}(p(\tau)) \nabla_{a}^{\gamma} \tau\right) \\
& =\exp \left(\int_{s}^{t} \widehat{\xi}_{v(\tau)}(p(\tau)) \widehat{\mathcal{G}}_{\gamma-1}\left(\sigma^{\gamma-1}(\tau), a\right) \nabla \tau\right) \\
& =\widehat{e}_{p}^{\gamma}(t, s ; a) \quad \text { if } a<s \leq t .
\end{aligned}
$$

(vi)

$$
\begin{aligned}
\widehat{e}_{p}^{\gamma}(t, a) \widehat{e}_{q}^{\gamma}(t, a) & =\exp \left(\int_{a}^{t}\left(\widehat{\xi}_{v(\tau)}(p(\tau))+\widehat{\xi}_{v(\tau)}(q(\tau)) \nabla_{a}^{\gamma} \tau\right)\right. \\
& =\exp \left(\int_{a}^{t} \widehat{\xi}_{v(\tau)}\left(\left(p \oplus_{v} q\right)(\tau)\right) \nabla_{a}^{\gamma} \tau\right) \\
& =\widehat{e}_{p \oplus v q}^{\gamma}(t, a) .
\end{aligned}
$$

(vi) The result follows easily using parts (iii) and (v).

Lemma 5.10 Let $g \in C_{\mathrm{ld}}^{\nabla}(\mathbb{T}), g(t)>0$ and $\frac{g^{\nabla}(t)}{g(t)} \in \mathcal{R}_{v}$. Then

$$
\nabla_{a}^{\gamma}[\log [g(t)]]=\widehat{\xi}_{\frac{\nu(t)}{\widehat{\mathcal{G}}_{1-\gamma}(t, a)}}\left[\frac{\nabla^{\gamma} g(t)}{g(t)}\right], \quad t>a \in \mathbb{T},
$$

where Log is the principle logarithm function. 
Proof Let $f(x)=\log [x]$. Clearly, $f: \mathbb{R}^{+} \rightarrow \mathbb{R}$ is continuous on $\mathbb{R}^{+}$. By using Lemma 3.6, we have

$$
\begin{aligned}
& \nabla_{a}^{\gamma}[\log [g(t)]]=\nabla_{a}^{\gamma}[(f \circ g)(t)] \\
& =\left\{\int_{0}^{1} f^{\prime}\left(g(t)-h v(t) g^{\nabla}(t)\right) d h\right\} \nabla_{a}^{\gamma} g(t) \\
& =\left\{\int_{0}^{1}\left[\frac{1}{g(t)-h v(t) g^{\nabla}(t)}\right] d h\right\} \nabla_{a}^{\gamma} g(t) \\
& =\left[-\frac{\log \left[g(t)-h v(t) g^{\nabla}(t)\right]}{v(t) g^{\nabla}(t)}\right]_{h=0}^{h=1} \widehat{\mathcal{G}}_{1-\gamma}(t, a) g^{\nabla}(t) \\
& =\left[-\frac{\log \left[g(t)-h v(t) g^{\nabla}(t)\right]}{v(t)}\right]_{h=0}^{h=1} \widehat{\mathcal{G}}_{1-\gamma}(t, a) \\
& = \begin{cases}-\frac{1}{\nu(t)} \log \left[\frac{g(t)-\nu(t) g^{\nabla}(t)}{g(t)}\right] \widehat{\mathcal{G}_{1-\gamma}}(t, a), & v(t) \neq 0 ; \\
\frac{g^{\nabla}(t)}{g(t)} \widehat{\mathcal{G}}_{1-\gamma}(t, a), & v(t)=0\end{cases} \\
& = \begin{cases}-\frac{\widehat{\mathcal{G}}_{1-\gamma}(t, a)}{\nu(t)} \log \left[1-v(t) \frac{g^{\nabla}(t)}{g(t)}\right], & v(t) \neq 0 ; \\
\frac{\nabla_{a}^{\gamma} g(t)}{g(t)}, & v(t)=0\end{cases} \\
& = \begin{cases}-\frac{\widehat{\mathcal{G}}_{1-\gamma}(t, a)}{\nu(t)} \log \left[1-\frac{v(t)}{\mathcal{G}_{1-\gamma}(t, a)} \frac{\nabla_{g}^{\gamma} g(t)}{g(t)}\right], & v(t) \neq 0 ; \\
\frac{\nabla_{a}^{\gamma} g(t)}{g(t)}, & v(t)=0\end{cases} \\
& =\widehat{\xi}_{\frac{v(t)}{\mathcal{G}_{1-\gamma}(t, a)}}\left[\frac{\nabla_{a}^{\gamma} g(t)}{g(t)}\right] \text {. }
\end{aligned}
$$

\section{Conformal dynamic equations and inequalities}

In this section, we consider solutions to the conformable dynamic equations and prove several related inequalities.

Let $f \in C_{\mathrm{ld}}\left([a, b]_{\mathbb{T}} \times \mathbb{R}\right)$ and $x \in C_{\mathrm{ld}}^{\nabla}\left([a, b]_{\mathbb{T}}\right)$. Consider the following conformable fractional dynamic equation:

$$
\nabla_{a}^{\gamma} x(t)=f(t, x(t)), \quad t \in[a, b]_{\mathbb{T}}, \gamma \in(0,1]
$$

However, one can also consider the integral form of the equation:

$$
x(t)=x(a)+\int_{a}^{t} \widehat{\mathcal{G}}_{\gamma-1}\left(\sigma^{\gamma-1}(\tau), a\right) f(\tau, x(\tau)) \nabla \tau .
$$

The integral form is useful for proving the existence and uniqueness of solutions or for studying analytical properties of solutions.

Lemma 6.1 Let $\gamma \in(0,1]$ and $f:[a, b]_{\mathbb{T}} \times \mathbb{R} \rightarrow \mathbb{R}$. Function $x \in C_{\mathrm{ld}}\left([a, b]_{\mathbb{T}}\right)$ is a solution of problem (6.1) if and only if $x$ is a solution of (6.2).

Proof $(\Rightarrow)$ By Theorem 4.10, we have

$$
\nabla_{a}^{-\gamma}\left(\nabla_{a}^{\gamma} x\right)(t)=\nabla_{a}^{-\gamma} f(t, x(t))
$$




$$
\begin{aligned}
& x(t)-x(a)=\int_{a}^{t} \widehat{\mathcal{G}}_{\gamma-1}\left(\sigma^{\gamma-1}(\tau), a\right) f(\tau, x(\tau)) \nabla \tau, \\
& x(t)=x(a)+\int_{a}^{t} \widehat{\mathcal{G}}_{\gamma-1}\left(\sigma^{\gamma-1}(\tau), a\right) f(\tau, x(\tau)) \nabla \tau .
\end{aligned}
$$

$(\Leftarrow)$ By taking $(\gamma, a)$-nabla derivative of both sides of (6.2), we have

$$
\begin{aligned}
\left(\nabla_{a}^{\gamma} x\right)(t) & =\nabla_{a}^{\gamma}\left[x(a)+\int_{a}^{t} \widehat{\mathcal{G}}_{\gamma-1}\left(\sigma^{\gamma-1}(\tau), a\right) f(\tau, x(\tau)) \nabla \tau\right] \\
& =\widehat{\mathcal{G}}_{1-\gamma}(t, a)\left[x(a)+\int_{a}^{t} \widehat{\mathcal{G}}_{\gamma-1}\left(\sigma^{\gamma-1}(\tau), a\right) f(\tau, x(\tau)) \nabla \tau\right]^{\nabla} \\
& =\widehat{\mathcal{G}}_{1-\gamma}(t, a)\left[\widehat{\mathcal{G}}_{\gamma-1}\left(\sigma^{\gamma-1}(t), a\right) f(t, x(t))\right] \\
& =f(t, x(t)) .
\end{aligned}
$$

These completes the proof.

Let $\mathbb{X}$ be the Banach space of all $x \in C_{\mathrm{ld}}\left([a, b]_{\mathbb{T}}\right)$ with the norm

$$
\|x\|:=\sup _{t \in[a, b]_{\mathbb{T}}}|x(t)|<\infty .
$$

In the following theorem, we examine the solution to problem (6.1) on Banach space $\mathbb{X}$.

Theorem 6.2 Let the function $f: \mathbb{X} \times \mathbb{R} \rightarrow \mathbb{R}$ be Lipschitz continuous with Lipschitz constant $L>0$. If

$$
\frac{L(t-a)^{\gamma}}{\gamma}<1, \quad t \in[a, b]_{\mathbb{T}}
$$

then problem (6.1) has a unique solution in $\mathbb{X}$.

Proof Define the operator $T: \mathbb{X} \rightarrow \mathbb{X}$ by

$$
T x(t)=x(a)+\int_{a}^{t} \widehat{\mathcal{G}}_{\gamma-1}\left(\sigma^{\gamma-1}(\tau), a\right) f(\tau, x(\tau)) \nabla \tau .
$$

We show that $T$ has a fixed point, which is a unique solution of $(6.1)$ on $[a, b]_{\mathbb{T}}$. For that, we show that $T$ is a contraction mapping on $\mathbb{X}$.

Now, for $x, y \in \mathbb{X}$, we have

$$
\begin{aligned}
|T x(t)-T y(t)| & \leq \int_{a}^{t} \widehat{\mathcal{G}}_{\gamma-1}\left(\sigma^{\gamma-1}(\tau), a\right)|f(t, x(t))-f(t, y(t))| \nabla \tau \\
& \leq L \sup _{t \in[a, b]_{\mathbb{T}}}|x(t)-y(t)| \int_{a}^{t} \widehat{\mathcal{G}}_{\gamma-1}\left(\sigma^{\gamma-1}(\tau), a\right) \nabla \tau \\
& \leq L \sup _{t \in[a, b]_{\mathbb{T}}}|x(t)-y(t)| \int_{a}^{t} \widehat{\mathcal{G}}_{\gamma-1}\left(\sigma^{\gamma-1}(\tau), a\right) \nabla \tau \\
& \leq L\|x-y\| \int_{a}^{t} \widehat{\mathcal{G}}_{\gamma-1}\left(\sigma^{\gamma-1}(\tau), a\right) \nabla \tau .
\end{aligned}
$$


Since for $\gamma \in(0,1), \gamma-1<0$, By Lemma 2.3 and Remark 2.6, we obtain that

$$
\int_{a}^{t} \widehat{\mathcal{G}}_{\gamma-1}\left(\sigma^{\gamma-1}(\tau), a\right) \nabla \tau \leq \int_{a}^{t}(\tau-a)^{\gamma-1} d \tau=\frac{(t-a)^{\gamma}}{\gamma}
$$

holds for all $t \in[a, b]_{\mathbb{T}}$.

It follows that

$$
\|T x-T y\| \leq \frac{L(t-a)^{\gamma}}{\gamma}\|x-y\|
$$

Thus, for $\frac{L(t-a)^{\gamma}}{\gamma}<1, T$ is a contraction mapping on $\mathbb{X}$. Hence, by Banach fixed point theorem, $T$ has a unique fixed point $x$ in $\mathbb{X}$. This completes the proof.

Considering the existence and uniqueness of the solution of the equation (6.1) above, by letting $f(t, y(t))=\Psi_{v}^{\gamma}(p(t) y(t))$, we are able to prove the following theorems.

Theorem 6.3 For $\gamma \in(0,1], a \in \mathbb{T}$ and $\Psi_{v}^{\gamma}(p) \in \mathcal{R}_{v}^{+}$, the exponential function $\widehat{e}_{p}^{\gamma}(t, a)$, defined by (5.3), is a unique solution of the following Cauchy problem:

$$
\nabla_{a}^{\gamma} y(t)=\Psi_{v}^{\gamma}(p(t)) y(t), \quad y(a)=1
$$

where

$$
\Psi_{\nu}^{\gamma}(p(t))=\frac{1-(1-v(t) p(t))^{\widehat{\mathcal{G}}_{\gamma-1}\left(\sigma^{\gamma-1}(t), a\right)}}{\nu(t) \widehat{\mathcal{G}}_{\gamma-1}\left(\sigma^{\gamma-1}(t), a\right)} \quad \text { and } \quad \Psi_{0}^{\gamma}(p(t))=p(t) \quad \text { for all } t \in \mathbb{T} \text {. }
$$

Proof It is sufficient to prove the property for left-scattered points (i.e., $\rho(t)<t)$. By the definition of conformable nabla derivative and part (ii) of Theorem 5.9, we have

$$
\begin{aligned}
\nabla_{a}^{\gamma} y(t) & =\frac{y(t)-y(\rho(t))}{\nu(t)} \widehat{\mathcal{G}}_{1-\gamma}(t, a) \\
& =\frac{\widehat{e}_{p}^{\gamma}(t, a)-\widehat{e}_{p}^{\gamma}(\rho(t), a)}{\nu(t)} \widehat{\mathcal{G}}_{1-\gamma}(t, a) \\
& =\frac{\left(1-(1-v(t) p(t))^{\widehat{\mathcal{G}}_{\gamma-1}\left(\sigma^{\gamma-1}(t), a\right)}\right)}{\nu(t) \widehat{\mathcal{G}}_{\gamma-1}\left(\sigma^{\gamma-1}(t), a\right)} \widehat{e}_{p}^{\gamma}(t, a) \\
& =\Psi_{v}^{\gamma}(p(t)) y(t),
\end{aligned}
$$

where

$$
\Psi_{\nu}^{\gamma}(p(t))=\frac{1-(1-v(t) p(t))^{\widehat{\mathcal{G}}_{\gamma-1}\left(\sigma^{\gamma-1}(t), a\right)}}{v(t) \widehat{\mathcal{G}}_{\gamma-1}\left(\sigma^{\gamma-1}(t), a\right)} \quad \text { for all } t \in \mathbb{T}
$$

and

$$
\Psi_{0}^{\gamma}(p(t))=\lim _{\nu \rightarrow 0} \Psi_{v}^{\gamma}(p(t))=p(t) \quad \text { for all } t \in \mathbb{T}
$$


Remark 6.4 Note that, if $\gamma=1$, we obtain the usual $\nabla$-derivative of the exponential function $\widehat{e}_{p}^{y}(t, a)$, i.e.,

$$
\nabla_{a}^{\gamma}\left[\widehat{e}_{p}^{\gamma}(t, a)\right]=\widehat{e}^{\nabla}(t, a)=p(t) \widehat{e}_{p}(t, a) .
$$

For $\mathbb{T}=\mathbb{R}$, we have $v(t)=0$ and $\Psi_{0}^{\gamma}(p(t))=p(t)$. Hence

$$
\nabla_{a}^{\gamma}\left[\widehat{e}_{p}^{\gamma}(t, a)\right]=p(t) e^{p \frac{(t-a)^{\gamma}}{\gamma}}
$$

The following lemma is useful.

Lemma 6.5 Let $\Psi_{v}^{\gamma}(p(t))$ be defined as in Theorem 6.3 above. Then

$$
\Psi_{\nu}^{\gamma}\left(\ominus_{\nu} p(t)\right)=\frac{-\Psi_{\nu}^{\gamma}(p(t))}{\left.(1-v(t) p(t))^{\hat{\mathcal{G}}_{\gamma-1}(\sigma \gamma-1}(t), a\right)} .
$$

Proof

$$
\begin{aligned}
\Psi_{\nu}^{\gamma}\left(\ominus_{\nu} p(t)\right) & =\frac{1-\left(1-v(t)\left(\ominus_{\nu} p\right)(t)\right)^{\widehat{\mathcal{G}}_{\gamma-1}\left(\sigma^{\gamma-1}(t), a\right)}}{\nu(t) \widehat{\mathcal{G}}_{\gamma-1}\left(\sigma^{\gamma-1}(t), a\right)} \\
& =\frac{1-(1-v(t) p(t))^{-\widehat{\mathcal{G}}_{\gamma-1}\left(\sigma^{\gamma-1}(t), a\right)}}{\nu(t) \widehat{\mathcal{G}}_{\gamma-1}\left(\sigma^{\gamma-1}(t), a\right)} \\
& =-\frac{1-(1-v(t) p(t))^{\widehat{\mathcal{G}}_{\gamma-1}\left(\sigma^{\gamma-1}(t), a\right)}}{v(t) \widehat{\mathcal{G}}_{\gamma-1}\left(\sigma^{\gamma-1}(t), a\right)} \cdot \frac{1}{(1-\nu(t) p(t))^{\widehat{\mathcal{G}}_{\gamma-1}\left(\sigma^{\gamma-1}(t), a\right)}} \\
& =-\frac{\Psi_{\nu}^{\gamma}(p(t))}{(1-v(t) p(t))^{\hat{\mathcal{G}}_{\gamma-1}\left(\sigma^{\gamma-1}(t), a\right)}} .
\end{aligned}
$$

Theorem 6.6 Suppose $p \in \mathcal{R}_{v}^{+}$. Let $\gamma \in(0,1], a \in \mathbb{T}$ and $y_{0} \in \mathbb{R}$. The unique solution of the initial value problem

$$
\nabla_{a}^{\gamma} y(t)=-\Psi_{v}^{\gamma}(p(t)) y(\rho(t)), \quad y(a)=y_{0}
$$

is given by

$$
y(t)=\widehat{e}_{\ominus \nu p}^{\gamma}(t, a) y_{0} .
$$

Proof Using Theorem 6.3 and Lemma 6.5, we get

$$
\begin{aligned}
\nabla_{a}^{\gamma} \widehat{e}_{\ominus_{v} p}^{\gamma}(t, a) & =\Psi_{v}^{\gamma}\left(\ominus_{\nu} p(t)\right) \widehat{e}_{\ominus_{v} p}^{\gamma}(t, a) \\
& =-\frac{\Psi_{v}^{\gamma}(p(t))}{(1-v(t) p(t))^{\widehat{\mathcal{G}}_{\gamma-1}\left(\sigma^{\gamma-1}(t), a\right)}} \widehat{e}_{\ominus_{\nu} p}^{\gamma}(t, a) \\
& =-\frac{\Psi_{\nu}^{\gamma}(p(t))}{(1-v(t) p(t))^{\widehat{\mathcal{G}}_{\gamma-1}\left(\sigma^{\gamma-1}(t), a\right)}} \cdot \frac{\widehat{e}_{\ominus_{\nu} p}^{\gamma}(\rho(t), a)}{\left(1-v(t)\left(\ominus_{\nu} p\right)(t)\right)^{\widehat{\mathcal{G}}_{\gamma-1}\left(\sigma^{\gamma-1}(t), a\right)}}
\end{aligned}
$$




$$
\begin{aligned}
& =-\frac{\Psi_{v}^{\gamma}(p(t))}{(1-v(t) p(t))^{\hat{\mathcal{G}}_{\gamma-1}\left(\sigma^{\gamma-1}(t), a\right)}} \cdot \frac{\widehat{e}_{\ominus_{v} p}^{\gamma}(\rho(t), a)}{(1-v(t) p(t))^{-\widehat{\mathcal{G}}_{\gamma-1}\left(\sigma^{\gamma-1}(t), a\right)}} \\
& =-\Psi_{v}^{\gamma}(p(t)) \widehat{\vec{e}}_{\ominus_{v} p}^{\gamma}(\rho(t), a) .
\end{aligned}
$$

Since $y(a)=\widehat{e}_{\ominus_{v} p}^{\gamma}(a, a) y_{0}=y_{0}$, we have the desired result.

Theorem 6.7 If $p \in \mathcal{R}_{v}^{+}$and $a, b, c \in \mathbb{T}$ are such that $a<b \leq c$, then

$$
\nabla_{a}^{\gamma}\left[\widehat{e}_{p}^{\gamma}(a, t)\right]=-\Psi_{v}^{\gamma}(p(t)) \widehat{e}_{p}^{\gamma}(a, \rho(t)), \quad t \in \mathbb{T}
$$

and

$$
\int_{b}^{c} \Psi_{v}^{\gamma}(p(t)) \widehat{e}_{p}^{\gamma}(a, \rho(t)) \nabla_{a}^{\gamma}=\widehat{e}_{p}^{\gamma}(a, b)-\widehat{e}_{p}^{\gamma}(a, c) .
$$

Proof We use Lemma 6.5 and the properties in Theorem 5.9 to find

$$
\begin{aligned}
& \Psi_{v}^{\gamma}(p(t)) \widehat{e}_{p}^{\gamma}(a, \rho(t)) \\
& =\Psi_{v}^{\gamma}(p(t)) \widehat{e}_{\ominus v}^{\gamma}(\rho(t), a) \\
& =\Psi_{v}^{\gamma}(p(t))\left(1-v(t)\left(\ominus_{v} p\right)(t)\right)^{\widehat{\mathcal{G}}_{\gamma-1}\left(\sigma^{\gamma-1}(t), a\right)} \widehat{e}_{\ominus_{v} p}^{\gamma}(t, a) \\
& =\frac{1-(1-v(t) p(t))^{\widehat{\mathcal{G}}_{\gamma-1}\left(\sigma^{\gamma-1}(t), a\right)}}{\nu(t) \widehat{\mathcal{G}}_{\gamma-1}\left(\sigma^{\gamma-1}(t), a\right)}\left(1-v(t)\left(\ominus_{\nu} p\right)(t)\right)^{\widehat{\mathcal{G}}_{\gamma-1}\left(\sigma^{\gamma-1}(t), a\right)} \widehat{\vec{e}}_{\ominus_{\nu} p}^{\gamma}(t, a) \\
& =-\frac{1-(1-v(t) p(t))^{-\widehat{\mathcal{G}}_{\gamma-1}\left(\sigma^{\gamma-1}(t), a\right)}}{\nu(t) \widehat{\mathcal{G}}_{\gamma-1}\left(\sigma^{\gamma-1}(t), a\right)} \widehat{e}_{\ominus v p}^{\gamma}(t, a) \\
& =-\Psi_{v}^{\gamma}\left(\ominus_{v} p(t)\right) \widehat{e}_{\ominus v}^{\gamma}(t, a) \\
& =-\nabla_{a}^{\gamma}\left[\widehat{e}_{\ominus v p}^{\gamma}(t, a)\right] \\
& =-\nabla_{a}^{\gamma}\left[\widehat{e}_{p}^{\gamma}(a, t)\right] \text {. }
\end{aligned}
$$

It follows that

$$
\begin{aligned}
\int_{b}^{c} \Psi_{v}^{\gamma}(p(t)) \widehat{e}_{p}^{\gamma}(a, \rho(t)) \nabla_{a}^{\gamma} t & =-\int_{b}^{c} \nabla_{a}^{\gamma}\left[\widehat{e}_{p}^{\gamma}(a, t)\right] \nabla_{a}^{\gamma} t \\
& =\widehat{e}_{p}^{\gamma}(a, b)-\widehat{e}_{p}^{\gamma}(a, c),
\end{aligned}
$$

which proves the desired identity.

For the introduction to the Gronwall's inequality, we refer to [34, 38]. The following dynamic inequality is useful in proving the Gronwall's inequality in this new setting.

Theorem 6.8 Let $y, f \in C_{\mathrm{ld}}(\mathbb{T}), p \in \mathcal{R}_{v}^{+}$and $a \in \mathbb{T}$. Then

$$
\nabla_{a}^{\gamma} y(t) \leq \Psi_{v}^{\gamma}(p(t)) y(t)+f(t) \quad \text { for all } t \in \mathbb{T}
$$

implies

$$
y(t) \leq y(a) \widehat{e}_{p}^{\gamma}(t, a)+\int_{a}^{t} \widehat{e}_{p}^{\gamma}(t, \rho(s) ; a) f(s) \nabla_{a}^{\gamma} s \quad \text { for all } t \in \mathbb{T} .
$$


Proof Using the product rule in Theorem 3.5 and Lemma 6.5, we get

$$
\begin{aligned}
\nabla_{a}^{\gamma}\left[y(t) \widehat{e}_{\ominus v p}^{\gamma}(t, a)\right] & =\nabla_{a}^{\gamma} y(t) \widehat{e}_{\ominus_{v} p}^{\gamma}(\rho(t), a)+y(t) \Psi_{v}^{\gamma}\left(\ominus_{v} p(t)\right) \widehat{e}_{\ominus_{v} p}^{\gamma}(t, a) \\
& =\nabla_{a}^{\gamma} y(t) \widehat{e}_{\ominus_{v} p}^{\gamma}(\rho(t), a)-\frac{y(t) \Psi_{v}^{\gamma}(p(t)) \widehat{e}_{\ominus_{v} p}^{\gamma}(t, a)}{(1-v(t) p(t))^{\hat{\mathcal{G}}_{\gamma-1}\left(\sigma^{\gamma-1}(t), a\right)}} .
\end{aligned}
$$

Note that, from Theorem 5.9(ii), we have

$$
\widehat{e}_{\ominus_{\nu} p}^{\gamma}(\rho(t), a)=\frac{\widehat{e}_{\ominus_{v} p}^{\gamma}(t, a)}{(1-v(t) p(t)))^{\hat{\mathcal{G}}_{\gamma-1}\left(\sigma^{\gamma-1}(t), a\right)}},
$$

which implies that

$$
\left.\widehat{e}_{\ominus v p}^{\gamma}(t, a)=\widehat{e}_{\ominus v p}^{\gamma}(\rho(t), a)(1-v(t) p(t))\right)^{\widehat{\mathcal{G}}_{\gamma-1}\left(\sigma^{\gamma-1}(t), a\right)} .
$$

It follows that

$$
\begin{aligned}
\nabla_{a}^{\gamma}\left[y(t) \widehat{e}_{\ominus_{v} p}^{\gamma}(t, a)\right] & =\nabla_{a}^{\gamma} y(t) \widehat{e}_{\ominus_{v} p}^{\gamma}(\rho(t), a)-\frac{y(t) \Psi_{v}^{\gamma}(p(t)) \widehat{e}_{\ominus_{v} p}^{\gamma}(t, a)}{(1-v(t) p(t)) \widehat{\mathcal{G}}_{\gamma-1}\left(\sigma^{\gamma-1}(t), a\right)} \\
& =\nabla_{a}^{\gamma} y(t) \widehat{e}_{\ominus_{v} p}^{\gamma}(\rho(t), a)-y(t) \Psi_{v}^{\gamma}(p(t)) \widehat{e}_{\ominus v}^{\gamma}(\rho(t), a) \\
& =\left[\nabla_{a}^{\gamma} y(t)-\Psi_{v}^{\gamma}(p(t)) y(t)\right] \widehat{e}_{\ominus_{v} p}^{\gamma}(\rho(t), a) \\
& \leq \widehat{e}_{\ominus_{\nu} p}^{\gamma}(\rho(t), a) f(t) .
\end{aligned}
$$

Now, taking $(\eta, a)$-integral on both sides of the latter inequality yields

$$
y(t) \widehat{e}_{\ominus_{\nu} p}^{\gamma}(t, a)-y(a) \leq \int_{a}^{t} \widehat{e}_{\ominus_{\nu} p}^{\gamma}(\rho(s), a) f(s) \nabla_{a}^{\gamma} s .
$$

Using parts (iii) and (v) of Theorem 5.9 yields

$$
\begin{aligned}
& y(t) \leq \widehat{e}_{p}^{\gamma}(t, a) y(a)+\widehat{e}_{p}^{\gamma}(t, a) \int_{a}^{t} \widehat{e}_{\ominus \nu p}^{\gamma}(\rho(s), a) f(s) \nabla_{a}^{\gamma} s, \\
& y(t) \leq \widehat{e}_{p}^{\gamma}(t, a) y(a)+\int_{a}^{t} \widehat{e}_{p}^{\gamma}(t, \rho(s), a) f(s) \nabla_{a}^{\gamma} s .
\end{aligned}
$$

This completes the proof.

Next, we prove Gronwall's inequality, which will be useful in establishing the stability of conformable dynamical systems on time scales.

Theorem 6.9 (Gronwall's inequality) Let $y, f \in C_{\mathrm{ld}}(\mathbb{T}), p \in \mathcal{R}_{v}^{+}$, and $a \in \mathbb{T}$. Then

$$
y(t) \leq f(t)+\int_{a}^{t} \Psi_{\nu}^{\gamma}(p(\tau)) y(\tau) \nabla_{a}^{\gamma} \tau
$$

implies

$$
y(t) \leq f(t)+\int_{a}^{t} \Psi_{v}^{\gamma}(p(\tau)) \widehat{e}_{p}^{\gamma}(t, \rho(\tau) ; a) f(\tau) \nabla_{a}^{\gamma} \tau .
$$


Proof Define $g(t)=\int_{a}^{t} \Psi_{v}^{\gamma}(p(\tau)) y(\tau) \nabla_{a}^{\gamma} \tau$. Then $g(a)=0$ and

$$
\nabla_{a}^{\gamma} g(t)=\Psi_{v}^{\gamma}(p(t)) y(t) \leq \Psi_{v}^{\gamma}(p(t)) f(t)+\Psi_{v}^{\gamma}(p(t)) g(t) .
$$

By Theorem 6.8,

$$
g(t) \leq \int_{a}^{t} \Psi_{v}^{\gamma}(p(\tau)) \widehat{e}_{p}^{\gamma}(t, \rho(\tau) ; a) f(\tau) \nabla_{a}^{\gamma} \tau .
$$

Since $y(t) \leq f(t)+g(t)$, the claim follows.

If we take $\mathbb{T}=h \mathbb{N}_{0}$ and $a=0$ in Gronwall's inequality, we obtain the following example.

Example 6.10 Let $\mathbb{T}=h \mathbb{Z} \cap[0, \infty)$. If $y, f: \mathbb{T} \rightarrow \mathbb{R}$ and $\eta>0$ is a constant such that

$$
y(t) \leq f(t)+\sum_{\tau \in(0, t / h]} \Psi_{v}^{\gamma}(\eta) y(\tau h) \quad \text { for all } t \in \mathbb{T}
$$

then

$$
y(t) \leq f(t)+\sum_{\tau \in(0, t h]} \Psi_{v}^{\gamma}(\eta) f(\tau h)\left(\frac{1}{1-\eta h}\right)^{\frac{t^{(\gamma)}-(\tau h-h)(\gamma)}{\gamma h}} \quad \text { for all } t \in \mathbb{T} .
$$

Corollary 6.11 Let $y \in C_{\mathrm{ld}}(\mathbb{T}), p \in \mathcal{R}_{v}^{+}, \lambda \in \mathbb{R}, a \in \mathbb{T}$ and $y \geq 0$. Then

$$
y(t) \leq \lambda+\int_{a}^{t} \Psi_{v}^{\gamma}(p(\tau)) y(\tau) \nabla_{a}^{\gamma} \tau
$$

implies

$$
y(t) \leq \lambda \widehat{e}_{p}^{\gamma}(t, a) \quad \text { for all } t \in \mathbb{T} .
$$

Proof By letting $f(t)=\lambda$ in Theorem 6.9, we have

$$
\begin{aligned}
y(t) & \leq \lambda+\int_{a}^{t} \lambda \Psi_{v}^{\gamma}(p(\tau)) \widehat{e}_{p}^{\gamma}(t, \rho(\tau) ; a) \nabla_{a}^{\gamma} \tau \\
& =\lambda\left[1+\int_{a}^{t} \Psi_{v}^{\gamma}(p(\tau)) \widehat{e}_{p}^{\gamma}(t, \rho(\tau) ; a) \nabla_{a}^{\gamma} \tau\right] \\
& =\lambda\left[1+\int_{a}^{t} \Psi_{v}^{\gamma}(p(\tau)) \widehat{e}_{p}^{\gamma}(t, \rho(\tau) ; a) \nabla_{a}^{\gamma} \tau\right] \\
& =\lambda\left[1+\widehat{e}_{p}^{\gamma}(t, a ; a)-\widehat{e}_{p}^{\gamma}(t, t ; a)\right] \\
& =\lambda \widehat{e}_{p}^{\gamma}(t, a),
\end{aligned}
$$

where we use Theorem 6.7 and $\widehat{e}_{p}^{\gamma}(t, a ; a)=\widehat{e}_{p}^{\gamma}(t, a)$. This completes the proof.

Finally, we present a new version of Gronwall's inequality in this new setting (see [38]). The proof of the following theorem is similar to that in [38]. 
Theorem 6.12 Let $y \in C_{\mathrm{ld}}(\mathbb{T}),-p(t) \in \mathcal{R}_{v}^{+}, \lambda \in \mathbb{R}, a \in \mathbb{T}$, and $y \geq 0$. Then

$$
y(t) \leq \lambda+\left|\int_{a}^{t} \Psi_{v}^{\gamma}(p(\tau)) y(\tau) \nabla_{a}^{\gamma} \tau\right|
$$

implies

$$
y(t) \leq \begin{cases}\lambda \widehat{e}_{p}^{\gamma}(t, a), & \text { for } t \in[a, \infty)_{\mathbb{T}} \\ \lambda \widehat{e}_{-p}^{\gamma}(t, a), & \text { for } t \in(-\infty, a]_{\mathbb{T}} .\end{cases}
$$

Proof The proof is given in two cases.

Case I. For $t \in[a, \infty)_{\mathbb{T}}$, we have

$$
y(t) \leq \lambda+\left|\int_{a}^{t} \Psi_{v}^{\gamma}(p(\tau)) y(\tau) \nabla_{a}^{\gamma} \tau\right|=\lambda+\int_{a}^{t} \Psi_{\nu}^{\gamma}(p(\tau)) y(\tau) \nabla_{a}^{\gamma} \tau .
$$

Then, by Corollary 6.11, we have

$$
y(t) \leq \lambda \widehat{e}_{p}^{\gamma}(t, a) \quad \text { for } t \in[a, \infty)_{\mathbb{T}} .
$$

Case II. For $t \in(-\infty, a]_{\mathbb{T}}$, let

$$
z(t)=\int_{a}^{t} \Psi_{v}^{\gamma}(p(\tau)) y(\tau) \nabla_{a}^{\gamma} \tau
$$

For any $s \in[t, a]_{\mathbb{T}}$, we have

$$
\begin{aligned}
y(t) & \leq \lambda+\left|\int_{a}^{s} \Psi_{v}^{\gamma}(p(\tau)) y(\tau) \nabla_{a}^{\gamma} \tau\right| \\
& =\lambda-\int_{a}^{s} \Psi_{v}^{\gamma}(p(\tau)) y(\tau) \nabla_{a}^{\gamma} \tau \\
& =\lambda-z(s) .
\end{aligned}
$$

Since, $y \geq 0, p \geq 0, \lambda>0$, we have $\lambda-z(s)>0$. It follows that

$$
\frac{y(s)}{\lambda-z(s)} \leq 1
$$

Multiplying by $-\Psi_{v}^{\gamma}(p(s))$ both sides of the above inequality, it follows that

$$
\begin{aligned}
& \frac{-\Psi_{v}^{\gamma}(p(s)) y(s)}{\lambda-z(s)} \geq-\Psi_{v}^{\gamma}(p(s)) \\
& \Rightarrow \quad \frac{\nabla_{a}^{\gamma}[\lambda-z(s)]}{\lambda-z(s)} \geq-\Psi_{v}^{\gamma}(p(s)) .
\end{aligned}
$$

Notice that

$$
\Psi_{v}^{\gamma}(p(s))=\frac{1-(1-v(s) p(s))^{\widehat{\mathcal{G}}_{\gamma-1}\left(\sigma^{\gamma-1}(s), a\right)}}{\nu(s) \widehat{\mathcal{G}}_{\gamma-1}\left(\sigma^{\gamma-1}(s), a\right)} \leq \frac{p(s)}{\widehat{\mathcal{G}}_{\gamma-1}\left(\sigma^{\gamma-1}(s), a\right)} .
$$


It follows that

$$
\frac{\nabla_{a}^{\gamma}[\lambda-z(s)]}{\lambda-z(s)} \geq-\Psi_{v}^{\gamma}(p(s)) \geq \frac{-p(s)}{\widehat{\mathcal{G}}_{\gamma-1}\left(\sigma^{\gamma-1}(s), a\right)} .
$$

Since $-p \in \mathcal{R}_{v}^{+}$, we have

$$
\frac{-p(s)}{\widehat{\mathcal{G}}_{\gamma-1}\left(\sigma^{\gamma-1}(s), a\right)} \leq \frac{\nabla_{a}^{\gamma}[\lambda-z(s)]}{\lambda-z(s)} \in \mathcal{R}_{\nu}^{+} .
$$

Integrating the above inequality over the interval $[t, a]_{\mathbb{T}}$ and using Lemma 5.10 leads to

$$
\begin{aligned}
& \int_{t}^{a} \widehat{\xi}_{\frac{\nu(s)}{\widehat{\mathcal{G}}_{1-\gamma}(s, a)}}\left[\frac{\nabla^{\gamma}[\lambda-z(s)]}{\lambda-z(s)}\right] \nabla^{\gamma} s \geq \int_{t}^{a} \widehat{\xi}_{\frac{\nu(s)}{\widehat{\mathcal{G}}_{1-\gamma}(s, a)}}\left[\frac{-p(s)}{\widehat{\mathcal{G}}_{\gamma-1}\left(\sigma^{\gamma-1}(s), a\right)}\right] \nabla^{\gamma} s, \\
& \left.\log [\lambda-z(s)]\right|_{t} ^{a} \geq \int_{t}^{a} \widehat{\xi}_{\frac{\nu(s)}{\widehat{\mathcal{G}}_{\left.1-\gamma^{(}, a\right)}}}\left[\frac{-p(s)}{\widehat{\mathcal{G}}_{\gamma-1}\left(\sigma^{\gamma-1}(s), a\right)}\right] \nabla^{\gamma} s, \\
& \log [\lambda]-\log [\lambda-z(t)] \geq \int_{t}^{a} \widehat{\xi}_{\frac{\nu(s)}{\widehat{\mathcal{G}}_{1-\gamma}(s, a)}}\left[\frac{-p(s)}{\widehat{\mathcal{G}}_{\gamma-1}\left(\sigma^{\gamma-1}(s), a\right)}\right] \nabla^{\gamma} s, \\
& \frac{\lambda}{\lambda-z(t)} \geq \exp \left[\int_{t}^{a} \widehat{\xi}_{\frac{\nu(s)}{\widehat{\mathcal{G}}_{1-\gamma}(s, a)}}\left[\frac{-p(s)}{\widehat{\mathcal{G}}_{\gamma-1}\left(\sigma^{\gamma-1}(s), a\right)}\right] \nabla^{\gamma} s\right] \text {, } \\
& \lambda-z(t) \leq \lambda \exp \left[-\int_{t}^{a} \widehat{\xi}_{\frac{v(s)}{\mathcal{G}_{1-\gamma}(s, a)}}\left[\frac{-p(s)}{\widehat{\mathcal{G}}_{\gamma-1}\left(\sigma^{\gamma-1}(s), a\right)}\right] \nabla^{\gamma} s\right], \\
& \lambda-z(t) \leq \lambda \exp \left[\int_{a}^{t} \widehat{\xi}_{v(s)}[-p(s)] \widehat{\mathcal{G}}_{1-\gamma}(s, a) \nabla^{\gamma} s\right] \leq \lambda \exp \left[\int_{a}^{t} \widehat{\xi}_{v(s)}[-p(s)] \nabla^{\gamma} s\right] \text {, } \\
& \lambda-z(t) \leq \lambda \widehat{e}_{-p}^{\gamma}(t, a) \quad \text { for } t \in[-\infty, a)_{\mathbb{T}} .
\end{aligned}
$$

Therefore,

$$
y(t) \leq \widehat{e}_{-p}^{\gamma}(t, a) \quad \text { for } t \in[-\infty, a)_{\mathbb{T}}
$$

This completes the proof.

\section{Concluding remarks}

In this paper we propose a new conformable nabla derivative and integral on arbitrary time scales which generalize the conformable fractional derivative and integral introduced in [5]. With this new definition of conformable derivative, we are able to define the conformable exponential function which is the solution to the linear conformable dynamic equation on time scales. Several useful results pertaining this exponential function are obtained. As an application, in the last section of this paper, we study the conformable dynamic equations and inequalities. We prove the Gronwall's inequality which is useful in establishing the stability of nabla conformable dynamical systems on time scales. 


\section{Funding}

None.

\section{Availability of data and materials}

Data sharing not applicable to this article as no datasets were generated or analyzed during the current study.

\section{Competing interests}

The authors declare that they have no competing interests.

\section{Authors' contributions}

The main idea of this paper was proposed by SR and MN. SR prepared the manuscript initially and performed all the steps of the proofs in this research. All authors read and approved the final manuscript.

\section{Author details}

1School of Mathematical Sciences, University of Nottingham Malaysia, Jalan Broga, Semenyih, Selangor Darul Ehsan 43500, Malaysia. ${ }^{2}$ School of Mathematical Science, Faculty of Science and Technology, University Kebangsaan Malaysia, Bangi, Selangor 43600, Malaysia.

\section{Publisher's Note}

Springer Nature remains neutral with regard to jurisdictional claims in published maps and institutional affiliations.

Received: 28 August 2020 Accepted: 19 April 2021 Published online: 03 May 2021

\section{References}

1. Daftardar-Gejji, V., Jafari, H.: Analysis of a system of nonautonomous fractional equations involving Caputo derivatives. J. Math. Anal. Appl. 328, 1026-1033 (2007)

2. Srivastava, H.M., Kilbas, A.A., Trujillo, J.J.: Theory and Applications of Fractional Differential Equations. Elsevier, New York (2006)

3. Podlubny, I.: Fractional Differential Equations. Academic Press, San Diego (1999)

4. Yousef, A., Khalil, R., Al Horani, M., Sababheh, M.: Analysis on measure chain-a unified approach to continuous and discrete calculus. Results Math. 18, 18-56 (1990)

5. Abdeljawad, T.: On conformable fractional calculus. J. Comput. Appl. Math. 279, 57-66 (2015)

6. Iyiola, O.S., Nwaezas, E.R.: Some new results on the new conformable fractional calculus with application using D'Alembert approach. Prog. Fract. Differ. Appl. 2, 1-7 (2016)

7. Kaabar, M.: Novel method for solving the conformable wave equation. J. New Theory 31, 56-85 (2020)

8. Kiskinov, H.: Remarks about the existence of conformable derivatives and some consequences

9. Ammi, M.R.S., Torres, D.F.S.: Existence of solution to a nonlocal conformable fractional thermistor problem. Commun. Fac. Sci. Univ. Ank. Sér. A1 Math. Stat. 68, 1060-1072 (2019)

10. Bendouma, B., Cabada, A., Hammoudi, A.: Existence results for systems of comformable fractional differential equations. Arch. Math. (Brno) 55, 69-82 (2019)

11. Thiramanus, P., Asawasamrit, S., Ntoutas, S.K., Tariboon, J.: Periodic boundary value problems for impulsive conformable fractional integro-differential equations. Bound. Value Probl. 2016, 122 (2016)

12. Eslami, M., Rezazadeh, $H .:$ The first integral method for Wu-Zhang system with conformable time-fractional derivative. Calcolo 53, 475-485 (2016)

13. Thabe, H., Kendre, S.: Analytical solutions for conformable space-time fractional partial differential equations via fractional differential transform. Chaos Solitons Fractals 109, 238-245 (2018)

14. Agarwal, P., Sitho, S., Ntouyas, S.K., Tariboon, J.: Noninstantaneous impulsive inequalities via conformable fractional calculus. J. Inequal. Appl. 2018, 261 (2018)

15. Ünal, E., Gökdogan, A., Çelik, E.: Solutions of sequential conformable fractional differential equations around an ordinary point and conformable fractional Hermite differential equation. Br. J. Appl. Sci. Technol. 10, 1-11 (2015)

16. Chung, W.S.: Fractional Newton mechanics with conformable fractional derivative. J. Comput. Appl. Math. 290, $150-158(2015)$

17. Aminikhah, H., Rezazadeh, H., Sheikhani, A.H.R.: Stability analysis of conformable fractional systems. Iran. J. Numer. Anal. Optim. 7(1), 13-32 (2017)

18. Mohammadnezhad, V., Eslami, M., Rezazadeh, H.: Stability analysis of linear conformable fractional differential equations system with time delays. Bol. Soc. Parana. Mat. 38, 159-171 (2020)

19. Anderson, D.R., Ulness, D.J.: Newly defined conformable derivatives. Adv. Dyn. Syst. Appl. 10, 109-137 (2015)

20. Abdelhakim, A.A.: The flaw in the conformable calculus: it is conformable because it is not fractional. Fract. Calc. Appl. Anal. 22(2), 242-254 (2019)

21. Abdelhakim, A.A., Machedo, J.A.T.: A critical analysis of the conformable derivative. Nonlinear Dyn. 95, 3063-3073 (2019)

22. Hassani, S., Benkhettou, N., Torres, D.F.M.: A conformable fractional calculus on arbitrary time scales. J. King Saud Univ. Sci. 28, 93-98 (2016)

23. Bendouma, B., Hammoudi, A.: A nabla conformable fractional calculus on time scales. Electron. J. Math. Anal. Appl. 7(1), 202-216 (2019)

24. Bohner, M., Hatipoğlu, V.F.: Dynamic cobweb models with conformable fractional derivatives. Nonlinear Anal. Hybrid Syst. 32, 157-167 (2019)

25. Nwaeze, E.R.: A mean value theorem for the conformable fractional calculus on arbitrary time scales. Prog. Fract. Differ. Appl. 2(4), 287-291 (2016)

26. Feng, Q., Meng, F.: Oscillation results for a fractional order dynamic equation on time scales with conformable fractional derivative. Adv. Differ. Equ. 2018, 193 (2018) 
27. Yilmaz, E., Gulsen, T., Goktas, S.: Conformable fractional Dirac system on time scales. J. Inequal. Appl. 2017, 161 (2017)

28. Rahmat, M.R.S.: A new definition of conformable fractional derivative on arbitrary time scales. Adv. Differ. Equ. 2019, 354 (2019)

29. Zhou, J., Wang, Y., Li, L.: Fractional Sobolev's spaces on time scales via conformable fractional calculus and their application to a fractional differential equation on time scales. Adv. Math. Phys. 2016, 9636491 (2016)

30. Zhao, D., Tongxing, L.: On conformable delta fractional calculus on time scales. J. Math. Comput. Sci. 16, 324-335 (2016)

31. Zhao, D.F., You, X.X.: Nabla local fractional derivative on time scales. Adv. Appl. Math. Anal. 11(1), $29-35$ (2016)

32. You, X.X., Zhao, D.F., Cheng, J.: Remarks on conformable fractional derivative on time scales. Adv. Theor. Appl. Math. $11(1), 61-68(2016)$

33. O'Regan, D., Agarwal, R.P., Bohner, M., Peterson, A.: Dynamic equations on time scales. J. Comput. Appl. Math. 141, $1-26$ (2002)

34. Bohner, M., Peterson, A.: Dynamic Equations on Time Scales: An Introduction with Applications. Birkhäuser, Boston (2001)

35. Bohner, M., Peterson, A.: Advances in Dynamic Equations on Time Scales. Birkhäuser, Boston (2003)

36. Ahmadkhanlu, A., Jahanshahi, M.: On the existence and uniqueness of solution of initial value problem for fractional order differential equations. Bull. Iran. Math. Soc. 38(1), 241-252 (2012)

37. Čermák, J., Nechvátal, L.: On (q,h)-analogue of fractional calculus. J. Nonlinear Math. Phys. 17(1), 51-68 (2010)

38. Lin, X.: A note on Gronwall's inequality on time scales. Abstr. Appl. Anal. 2014, 623726 (2014)

\section{Submit your manuscript to a SpringerOpen ${ }^{\circ}$ journal and benefit from:}

- Convenient online submission

Rigorous peer review

- Open access: articles freely available online

- High visibility within the field

- Retaining the copyright to your article

Submit your next manuscript at $\gg$ springeropen.com 